NBER WORKING PAPER SERIES

\title{
DEMAND HETEROGENEITY IN INSURANCE MARKETS: IMPLICATIONS FOR EQUITY AND EFFICIENCY
}

\author{
Michael Geruso \\ Working Paper 22440 \\ http://www.nber.org/papers/w22440 \\ NATIONAL BUREAU OF ECONOMIC RESEARCH \\ 1050 Massachusetts Avenue \\ Cambridge, MA 02138 \\ July 2016
}

I thank seminar and conference participants at ASHEcon, the BU/Harvard/MIT Health Economics Seminar, Duke, the University of Maryland, Princeton, the Robert Wood Johnson Foundation, and the University of Texas at Austin for useful comments, as well as Janet Currie, Angus Deaton, Josephine Duh, Ben Handel, Bo Honoré, Scott Kostyshak, Penka Kovacheva, Yan Lau, Timothy Layton, John Papp, Zhuan Pei, Uwe Reinhardt, Andrew Robinson, Harvey Rosen, Andrew Shephard, Dean Spears, Steve Trejo, Tom Vogl, and Lisa Vura-Weis for useful feedback. I especially thank my advisers Anne Case and David Lee for their guidance, and I gratefully acknowledge financial support from the Robert Wood Johnson Foundation and from grants 5 R24 HD042849 and 5 T32 HD007081 awarded to the Population Research Center at the University of Texas at Austin by the Eunice Kennedy Shriver National Institute of Child Health and Human Development. The views expressed herein are those of the author and do not necessarily reflect the views of the National Bureau of Economic Research.

NBER working papers are circulated for discussion and comment purposes. They have not been peer-reviewed or been subject to the review by the NBER Board of Directors that accompanies official NBER publications.

(C) 2016 by Michael Geruso. All rights reserved. Short sections of text, not to exceed two paragraphs, may be quoted without explicit permission provided that full credit, including () notice, is given to the source. 
Demand Heterogeneity in Insurance Markets: Implications for Equity and Efficiency

Michael Geruso

NBER Working Paper No. 22440

July 2016, Revised January 2017

JEL No. I11,I13

\begin{abstract}
In many markets insurers are barred from price discrimination based on consumer characteristics like age, gender, and medical history. In this paper, I build on a recent literature to show why such policies are inefficient if consumers differ in their willingness-to-pay for insurance conditional on the insured losses they generate. Using administrative claims data, I then show that this type of demand heterogeneity is empirically relevant in a consumer health plan setting. Younger and older consumers and men and women reveal strikingly different demand for health insurance, conditional on their objective medical spending risk. This implies that these groups must face different prices in order to sort themselves efficiently across insurance contracts. The theoretical and empirical analysis highlights a fundamental tradeoff between equity and efficiency that is unique to selection markets.
\end{abstract}

Michael Geruso

University of Texas at Austin

Department of Economics

1 University Station C3100

Austin, TX 78712

and NBER

mike.geruso@austin.utexas.edu 
In many insurance markets in the US and abroad, insurers face restrictions against setting premiums based on observable consumer characteristics like age, sex, and claims history. For employersponsored health plans in the US, such restrictions have been in place since $1974 .{ }^{1}$ In recent years, these types of non-discrimination rules have become more widely adopted across insurance market settings. For example, plans in the Affordable Care Act (ACA) Marketplaces cannot price-discriminate on sex or medical history, and there are binding restrictions on how age can enter pricing. In the same spirit, the European Union's high court ruled in 2011 that sex cannot enter premium determination in health insurance, life insurance, or annuities, even though sex is a strong predictor of insurer costs in these markets.

These types of regulations, aimed at promoting equity, are well known to exacerbate asymmetric information problems. This is because even though insurers can easily observe consumer characteristics that predict costs, they are constrained by regulators to act as if these characteristics were unobservable in premium-setting. This leaves markets susceptible to unravelling as more costly consumers select into more generous contracts (Akerlof, 1970). For this reason, non-discrimination policies are generally accompanied by complementary regulations like consumer tax subsidies and insurer subsidies in the form of risk adjustment. ${ }^{2}$ In the ACA Marketplaces, Medicare Advantage, Medicare Part D, and insurance markets around the world, these policy tools are used to counteract selection distortions that uniform pricing would otherwise introduce. The conventional wisdom is that with the right subsidies, uniform pricing carries no efficiency cost relative to any other feasible pricing policy. ${ }^{3}$ The view is supported by much of the early literature in risk adjustment, including Cutler and Reber (1998), Van de Ven and Ellis (2000), and Glazer and McGuire (2000). It is also reflected in the Chetty and Finkelstein (2013) handbook chapter on social insurance and in the "textbook" selection framework of Einav and Finkelstein (2011) that has become a widely applied guide for empirical work.

In this paper, I show why this conventional wisdom is wrong. In selection markets, if con-

\footnotetext{
${ }^{1}$ The Employee Retirement Income Security Act of 1974 (ERISA), amended by the Health Insurance Portability and Accountability Act of 1996 (HIPAA), rule-out distinctions in health benefits coverage made on the basis of an employee's or dependent's sex, race, age, national origin, religion, or disability, as well as health status or genetic information.

${ }^{2}$ Risk adjustment consists of a subsidy schedule that attempts to make all consumers appear equally costly to the insurer, on net and in expectation. Insurers are subsidized for enrolling-high expected-cost consumers and taxed for enrolling lowexpected-cost consumers, weakening insurer incentives to engage in cream-skimming, the Rothschild and Stiglitz (1976) distortion. By flattening the insurer's net cost curve, risk adjustment also addresses the Akerlof (1970) unravelling problem.

${ }^{3}$ Feasible here means subject to the constraint that costs are unobservable and/or cannot be used in pricing. Following Einav, Finkelstein and Cullen (2010) and others, the notion of constrained efficiency throughout the paper assumes that prices and subsidies are the only available policy instruments: A planner cannot directly allocate consumers to plans.
} 
sumers differ in their valuations of insurance contracts conditional on the costs they generate for insurers, then discriminatory pricing can represent a feasible welfare improvement over the best non-discriminatory pricing. The intuition is straightforward: Efficient prices are determined by the intersection of the willingness-to-pay and marginal cost curves. Therefore, if groups of buyers like men and women, rich and poor, or young and old have systematically different willingness-to-pay for insurance holding expected losses (i.e., insurer marginal costs) fixed, then the prices that would induce efficient self-sorting must be different across these groups. ${ }^{4}$ This is a unique feature of selection markets. Unlike other consumer goods, the producer's marginal cost of generating an insurance contract is fundamentally tied to the characteristics of the particular consumer who purchases the contract. The consumers generate both the demand and cost curves.

Under what conditions would this kind of demand heterogeneity exist? For one, consumers that represent the same actuarial risk to insurers may simply differ in their attitudes toward risk and therefore willingness-to-pay for insurance (Finkelstein and McGarry, 2006; Cohen and Einav, 2007; Fang, Keane and Silverman, 2008). Alternatively, consumers with identical risk preferences that face the same expected losses may nonetheless differ in the spread of their risk distributions, a possibility often assumed away in the simple foundational models like Rothschild and Stiglitz (1976) that consider a binary insurable loss. Or, even assuming identical risk preferences and identical insurable risk profiles, if utility does not take the constant absolute risk aversion (CARA) form, differences in wealth will drive differences in willingness-to-pay for insurance. ${ }^{5}$ The model of this paper nests these and many other potential drivers of demand heterogeneity that are ignored under the conventional notion of efficiency in insurance markets, because that notion implicitly assumes a one-to-one mapping from insurable risk to insurance valuation.

I begin in the paper by adapting the canonical model of insurance choice to accommodate optimal insurance pricing under heterogenous demand. The main theoretical result is that when demand differs across observable consumer groups, so too must prices in order to achieve the best feasible sorting of consumers into contracts. The intuition behind the result is illustrated in a series of simple

\footnotetext{
${ }^{4}$ In the presence of demand heterogeneity, marginal costs no longer trace a single curve but rather become a cloud of points. Because there is no unique intersection point between this cloud and the demand curve, there is no uniform price that generates efficient consumption.

${ }^{5}$ Even assuming identical insurable losses, identical risk preferences, and identical CARA utility, consumers with different wealth would display differential demand for insurance if bankruptcy protections limited the downside exposure for consumers with little wealth (Mahoney, 2015). In addition, Ericson and Starc (2015) provide some direct evidence of heterogenous price sensitivity in insurance markets across identifiable groups of consumers.
} 
figures, extending the now-standard graphical selection frameworks in Cutler and Reber (1998), Feldman and Dowd (2000), Einav and Finkelstein (2011), and Hackmann, Kolstad and Kowalski (2015). I then develop a simple sufficient statistics test to detect the relevant kind of demand heterogeneity. This test has minimal data and identification requirements and can be implemented broadly: The econometrician or regulator need observe only equilibrium plan choices and claims data.

I demonstrate the empirical relevance of the model using detailed administrative health claims data from a large employer, which allow precise controls for expected and realized healthcare spending. These data show that there is substantial demand heterogeneity across easily identifiable demographic groups. Willingness-to-pay for the more generous insurance option offered is strongly correlated with age and sex, even after conditioning on medical spending risk. For instance, 50- to 59 -year-old workers in this setting are 50\% more likely than 18- to 29-year-old workers to choose more insurance, holding expected medical spending fixed at any level. The theoretical framework of the paper makes it clear that these facts alone-without additional identifying assumptions-are sufficient to indicate that implementing age-specific pricing would yield a welfare improvement. ${ }^{6}$ The demand patterns, which are revealed in simple semi-parametric plots of plan choices versus administrative claims costs, stand in stark contrast to conventional wisdom, which would imply that young and old would differ in demand only because they differed in insurable risk, and which would predict that takeup conditional on insurable risk would be identical across the two groups.

Despite significant theoretical and empirical research attention to selection in recent years, the implications of heterogeneous demand in selection markets has been relatively unexplored. Only a small prior literature has recognized that first-best allocations cannot be achieved under asymmetric information with demand heterogeneity: Einav and Finkelstein (2011) note the phenomenon, and Glazer and McGuire (2011) and Bundorf, Levin and Mahoney (2012) address it in more depth, with the latter paper establishing the general result that a first-best is infeasible if demand heterogeneity exists. ${ }^{7}$ But with the sole exception of a stylized treatment in Glazer and McGuire (2011), past studies

\footnotetext{
${ }^{6}$ Age and sex are not assumed to be the underlying causes of the demand heterogeneity, but rather merely correlated with unobserved demand shifters. This is nonetheless sufficient to indicate welfare gains from optimal discriminatory pricing relative to the optimal uniform price. The model of Section 2 and empirical framework in Section 3 make this point formally and in detail.

${ }^{7}$ Einav and Finkelstein (2011) note: "Another important (and more nuanced) aspect of preference heterogeneity is that it complicates the notion of efficiency. With preference heterogeneity, the mapping from expected cost to willingness to pay need no longer be unique. That is, two individuals with the same expected cost may have different valuations for the same coverage, or two individual with the same willingness to pay for the coverage may have different underlying expected costs."
} 
have only considered market segmentation according to consumer cost types. ${ }^{8}$ The key innovation of my paper is to show that although a first-best is infeasible, there is a feasible welfare improvement over non-discriminatory pricing that can be achieved by segmenting the market according to consumer preference types. Importantly, I show that the result holds even in the extreme (but conceptually simple) case of segmenting two groups that have different preferences but identical costs.

To complement the sufficient statistics approach that constitutes the main empirical analysis, I also adapt a standard (e.g., Handel, 2013; Handel, Kolstad and Spinnewijn, 2015) expected utility model of insurance choice. The additional structure allows me to (i) show that the data matches the model in terms of across- and within-group demand heterogeneity; (ii) show that constrained optimal prices vary across demographic groups; and (iii) estimate the misallocation of consumers across plans due to non-discrimination policies. I find that older consumers and women optimally face higher premiums, and as a result younger consumers and men are sub-optimally underinsured when facing (constrained) optimal uniform prices. The unique insight of this paper is that optimal pricing differs not because these groups face different health spending risk, which they do. With standard regulatory tools like subsidies to counteract unravelling, uniform prices could sort all consumers perfectly. Rather, optimal prices differ because, on average, consumers in these groups value insurance contracts differently when they face the same risk.

The general theoretical phenomenon I describe is likely to be broadly relevant. Empirical work across a variety of insurance market settings has shown that consumer demand for insurance can deviate significantly from the underlying insurable risk. Cutler, Finkelstein and McGarry (2008) provide a survey of this literature and suggest that, "heterogeneity in preferences may be as, or more, important than heterogeneity in risk in explaining insurance demand." The prior research has almost exclusively focused on preference heterogeneity that is correlated with risk (positively or negatively). The present paper advances this literature in a new direction, showing why the part of demand heterogeneity that is uncorrelated with risk also has important implications in these markets. This is the key departure from prior work.

The central finding of this paper-that a social planner setting prices in insurance markets would

\footnotetext{
${ }^{8}$ Einav and Finkelstein (2011) do not consider the possibility of preference-based pricing. Bundorf, Levin and Mahoney (2012), one of the closest prior papers, shows that when willingness-to-pay varies among consumers carrying the same objective risk, a first-best allocation is infeasible without prices set at individual-specific marginal costs-in other words, without assuming away the problem of asymmetric information. Only one prior paper, Glazer and McGuire (2011), considers a policy that conditions insurance prices on some measure of a consumer's willingness-to-pay. That paper presents a stylized model of income-based premiums in an Exchange setting, but offers no empirical application.
} 
want to set different prices for different groups of consumers-also contrasts sharply with current policy in the US and abroad, which has trended toward non-discriminatory prices. While regulators and policymakers may nonetheless wish to pursue equity objectives, this paper makes clear that there is an unavoidable efficiency cost of doing so. ${ }^{9}$ Subsequent work that builds on the insight of the present paper, including Layton, Ellis and McGuire (2015), has begun to take this explicitly into account. ${ }^{10}$ The findings here also highlight the loss inherent in using insurance price regulation as a redistributive mechanism (Finkelstein, Poterba and Rothschild, 2009).

The rest of the paper proceeds as follows. Section 2 models the relevant phenomenon, then develops the novel insights regarding price discrimination. This section also outlines the sufficient statistic test. Section 3 describes the data, and Section 4 presents a set of reduced form empirical results that demonstrate that willingness-to-pay for insurance varies systematically with age and sex for reasons unrelated to health spending. Section 5 estimates a structural model of plan choice that builds on the basic reduced-form findings. Finally, in Section 6, the structural parameter estimates are used to infer the welfare impacts of alternative pricing policies that allow for discrimination.

\section{Demand Heterogeneity in Insurance Markets}

In this section I highlight the efficiency problem created by demand heterogeneity, within the context of the standard model of insurance market selection. I then develop the novel result: that a social planner setting prices (or a regulator setting subsidies) can improve welfare by setting prices that are a function of some observable characteristic that is correlated with revealed consumer preferences. This holds even if that characteristic is not correlated with the insurable risk. Finally, I develop a simple empirical test that uses commonly available claims data to reveal whether pricing-relevant demand heterogeneity exists in a market.

\footnotetext{
${ }^{9}$ The perspective of this paper is one of static efficiency, which follows most of the prior literature and permits a focus on the heterogeneity phenomenon of interest. Dynamic efficiency under uniform pricing is more complex to assess due to reclassification risk (Handel, Hendel and Whinston, 2015). The dynamic efficiency issue might be of secondary importance in this setting in any case: Age-based price discrimination is the primary empirical focus below, and as Handel, Hendel and Whinston (2015) note, there is no reclassification risk associated with the deterministic process of aging.

${ }^{10} \mathrm{My}$ study also connects to the broader body of recent empirical work estimating selection and efficiency in US insurance markets (e.g., Einav, Finkelstein and Cullen, 2010 and Hackmann, Kolstad and Kowalski, 2015). Such studies follow the earlier foundational work by Cutler and Reber (1998), Glazer and McGuire (2000), and others in abstracting away from consumer preference heterogeneity that is uncorrelated with insurer costs. While the approach of such papers is appropriate for considering welfare losses due to selection under current policy constraints, the model of this paper illustrates the possibility of feasible policy alternatives with additional potential for welfare gains.
} 


\subsection{The Canonical Model}

Consider consumers $i \in I$, who are described by characteristics partitioned into two vectors, $\delta$ and $\psi$. The vector $\delta$ describes any consumer characteristics that affect the insurer's costs of providing insurance, including the consumer's health risk. $\psi$ contains all other consumer characteristics relevant for insurance choice, such as wealth and risk preferences. Insurance contracts $j \in J$ are described by prices $p$ and plan features $\phi$, where $\phi$ includes networks, copays, deductibles, etc.

Uncertain future health expenditures are made in various states of the world $s \in S$, where the probability distribution over health states for person $i$ is $G_{i}=G\left(s \mid \delta_{i}\right)$. The expected utility of a contract $\left(p_{j}, \phi_{j}\right)$ to a consumer $\left(\delta_{i}, \psi_{i}\right)$ is:

$$
v\left(p_{j}, \phi_{j}, \delta_{i}, \psi_{i}\right)=\int u\left(s, p_{j}, \phi_{j}, \delta_{i}, \psi_{i}\right) \cdot G\left(s \mid \delta_{i}\right) d s
$$

This setup is intended to closely track the "canonical model" of insurance choice in Einav, Finkelstein and Levin (2010). ${ }^{11}$ With expected utility defined as above, consumers choose plans that generate the highest expected utility:

$$
v\left(p_{j}, \phi_{j}, \delta_{i}, \psi_{i}\right) \geq v\left(p_{k}, \phi_{k}, \delta_{i}, \psi_{i}\right) \forall k \in J
$$

Risk-neutral insurers incur costs due to claims paid to providers. Expected costs to the insurer, $c_{i j}$, depend on state-specific health events and plan characteristics like deductibles:

$$
c\left(\phi_{j}, \delta_{i}\right)=\int \tau\left(s, \phi_{j}, \delta_{i}\right) \cdot G\left(s \mid \delta_{i}\right) d s,
$$

where $\tau$ expresses state-specific insurer costs. Note that the insurer's expected costs depend on consumer characteristics that determine the distribution of health risk $G\left(s \mid \delta_{i}\right)$ but not on preferences or wealth, which are contained in $\psi_{i}$.

Define total social surplus as $W=\sum_{i \in I} \sum_{j \in J} I\left(j_{i}\right) \cdot\left(v\left(p_{j}, \phi_{j}, \delta_{i}, \psi_{i}\right)-c\left(\phi_{j}, \delta_{i}\right)\right)$, where $I\left(j_{i}\right)$ is an indicator function for person $i$ being enrolled in plan $j$. Maximizing $W$ requires that consumers sort to

\footnotetext{
${ }^{11}$ Compared to Einav, Finkelstein and Levin (2010), there are several differences here: The risk distribution here $(G(s \mid \delta))$ is continuous rather than discreet, consumer characteristics here are partitioned into two vectors $(\delta$ and $\psi)$ rather than one to highlight that preferences and constraints enter valuations but not costs, and the setup here ignores moral hazard in order to focus on the selection issues that are the primary interest of the paper. I nonetheless discuss the implications of moral hazard for the empirical exercise. See Section 4.2.
} 
plans where their valuation is in the greatest excess of the cost of providing insurance:

$$
v\left(p_{j}, \phi_{j}, \delta_{i}, \psi_{i}\right)-c\left(\phi_{j}, \delta_{i}\right) \geq v\left(p_{k}, \phi_{k}, \delta_{i}, \psi_{i}\right)-c\left(\phi_{k}, \delta_{i}\right) \forall k \in J
$$

This is the standard efficiency condition. It is typically invoked to illustrate how in markets with adverse selection, competitive equilibria are inefficient without regulatory interventions, such as consumer or insurer subsidies.

\subsection{Preference Heterogeneity in the Canonical Model}

Without departing from the canonical model, it is straightforward to observe that the surplus generated by a plan choice can differ between individuals $i$ and $i^{\prime}$ who would generate the same expected cost to insure. In particular, the preference parameter $\psi$ enters $v$ but not $c$, so that $c\left(\phi_{j}, \delta_{i}\right)=c\left(\phi_{j}, \delta_{i^{\prime}}\right)$ does not imply $v\left(p_{j}, \phi_{j}, \delta_{i}, \psi_{i}\right)=v\left(p_{j}, \phi_{j}, \delta_{i^{\prime}}, \psi_{i^{\prime}}\right)$. In other words, there is no one-to-one mapping between the $v$ and $c$ functions.

It is not difficult to conceive of quantitatively important demand heterogeneity remaining after conditioning on expected costs. Higher risk aversion, captured in $\psi$, will correspond to higher willingness-to-pay for insurance, holding fixed the actual risk distribution that a consumer faces. There is indeed substantial empirical evidence of heterogeneity in preferences over insurance purchase (e.g., Finkelstein and McGarry, 2006; Cohen and Einav, 2007; Fang, Keane and Silverman, 2008; Cutler, Finkelstein and McGarry, 2008). The conceptual framework also nests other drivers of variation in willingness-to-pay for insurance, holding $c_{i j}$ fixed. For instance, if risk preferences are identical across consumers but utility does not take the constant absolute risk aversion (CARA) form commonly assumed in empirical insurance studies, then differences in income will drive heterogeneity in $v$ conditional on $c$. Similarly, if assets at risk vary, for example due to bankruptcy laws that act as implicit insurance (Mahoney, 2015), then so too will consumers' valuation of an insurance contract, even when faced with identical preferences and identical health risk, $G(s)$, and when such consumers would generate identical costs to the insurer.

Alternatively, even in the case when both preferences $\psi$ and expected insurer losses $c_{i j}$ are identical between consumers, valuations can nonetheless differ if the risk distributions, $G$, differ in higher

moments. If $\widetilde{G}$ is a mean-preserving spread of $G$, then an otherwise similar consumer facing $\widetilde{G}$ will 
value insurance more highly than a consumer facing $G$. This is true even though expected insurer (and social resource) costs are identical for the $\widetilde{G}$ and $G$ cases.

In general, the welfare-maximizing insurance allocation may differ across consumers among whom $c$ is equal for a variety of plausible reasons. The sufficient statistics test that follows in Section 2.5 is intended to capture any such reason.

\subsection{Implications for Efficient Sorting}

Figures 1 and 2 illustrate the important interplay of selection and demand heterogeneity in a series of simple plots. Before illustrating in Figure 2 how selection and demand heterogeneity interact, I first reference in Figure 1 two familiar baseline cases: heterogeneity without selection in Panel A, and selection without heterogeneity in Panel B.

The top panels of Figure 1 plot joint distributions of $v\left(\phi_{j}, \delta_{i}, \psi_{i}\right)$ and $c\left(\phi_{j}, \delta_{i}\right)$ associated with some insurance contract $j$. Valuations, abbreviated $v_{i}$, are along the vertical axes, and expected costs, $c_{i}$, are along the horizontal axes. The valuations and costs are relative to an outside option, which can be assumed to be no insurance. ${ }^{12}$ For simplicity, each point in these plots can be interpreted as representing the contract valuation and expected cost pair of an individual consumer. ${ }^{13}$ The heterogeneity of interest occurs when $v_{i}$ varies across individuals, holding $c_{i}$ fixed.

A consumer purchases the contract if and only if $v_{i} \geq p$. A price $p^{*}$ is efficient if it satisfies $v_{i} \geq p^{*}$ if and only if $v_{i} \geq c_{i}$. In the figure, a dashed 45-degree line separates the space into efficient contracting above the 45-degree line $\left(v_{i} \geq c_{i}\right)$ and efficient non-contracting below it $\left(v_{i}<c_{i}\right)$. Horizontal lines in Figure 1 correspond to prices that induce consumers to self-sort into efficient choices.

Panel A corresponds to a typical goods market. There is heterogenous willingness-to-pay, leading to downward sloping demand, but there is no inherent relationship between $v_{i}$ and $c_{i}$. Panel C

\footnotetext{
${ }^{12}$ For expositional simplicity, I choose joint distributions of $(v, c)$ that yield linear demand curves, but the intuition applies more generally. Also for simplicity, I discuss the figure as representing a choice between purchasing a single available contract and no purchase, but the figure is consistent with a multi-contract setting after a simple transformation. To transform the multi-contract case into the same terms, define $k *$ as the plan generating the second highest surplus within the choice set. Then rearrange the efficiency condition $v\left(p_{j}, \phi_{j}, \delta, \psi\right)-c\left(\phi_{j}, \delta\right) \geq v\left(p_{k *}, \phi_{k *}, \delta, \psi\right)-c\left(\phi_{k *}, \delta\right)$ as $\left(v\left(p_{j}, \phi_{j}, \delta, \psi\right)-v\left(p_{k *}, \phi_{k *}, \delta, \psi\right)\right) \geq\left(c\left(\phi_{j}, \delta\right)-c\left(\phi_{k *}, \delta\right)\right)$, and finally redefine $\widetilde{v}$ and $\widetilde{c}$ as these differences to get the scatterpoints $\left(\widetilde{v_{i}}, \widetilde{c_{i}}\right)$ and the efficient takeup condition $\widetilde{v_{i}} \geq \widetilde{c_{i}}$. Finally, in these plots I make the standard assumption that price is separable from other aspects of insurance valuation-that is, I write $v_{i}$ to represent $v\left(\phi_{j}, \delta_{i}, \psi_{i}\right)$, rather than $v\left(p_{j}, \phi_{j}, \delta_{i}, \psi_{i}\right)$. A more general formulation would require three-dimensional plots to accommodate changes in $v_{i}$ with prices. The plots in Figure 1 and can be viewed as two-dimensional slices from such plots for some fixed price.

${ }^{13}$ Alternatively, the figures can be interpreted as plotting a measure of consumers, with scatter points within each panel representing the preferences and expected costs of point masses of consumers.
} 
plots the corresponding demand diagram. Note that the apparent uniformity of marginal costs in Panel A of Figure 1 is not intended to rule out non-constant marginal costs at the industry or firm level, but it does assume that the particular consumer purchasing the contract does not affect the producer's marginal costs. ${ }^{14}$

Panel B of Figure 1 corresponds to the framework most widely applied to guide empirical work on selection. It is constructed to generate the same demand curve as in Panel A, for ease of comparison. In contrast to panel A, there is no demand heterogeneity, but there is selection: Costs in Panel B are systematically related to valuations. This represents the special feature of insurance markets with asymmetric information: The firm's costs of producing the good are a function of the characteristics of consumers purchasing the goods and are therefore linked to consumers' valuations. In particular, the joint distribution is constructed here such that higher valuations are associated with higher costs, generating adverse selection on price. In the corresponding demand diagram in Panel D, this selection generates downward sloping demand and marginal cost curves. Panel D of Figure 1 mirrors the graphical frameworks in Einav, Finkelstein and Cullen (2010), Hackmann, Kolstad and Kowalski (2015), and other recent studies, which in turn echo Cutler and Reber (1998) and Feldman and Dowd (2000) and ultimately stylize the intuition of the Akerlof (1970) lemons model.

Much of the recent empirical literature on selection in insurance markets has built on the intuition embodied in Panel D to demonstrate the extent to which perfect competition, which generates average cost pricing, induces inefficient consumer sorting or market unravelling, or in the case of Starc (2014), that imperfect competition can act as a countervailing force against this unravelling. One important takeaway from this "textbook" selection model is that while inefficient average cost pricing naturally occurs in competitive markets, proper interventions (such as consumer subsidies) can induce efficient sorting in competitive markets without sacrificing the equity goal of making premiums independent of health state, age, or gender. ${ }^{15}$

Because the intention is to show the conditions under which prices can induce efficient sorting of consumers, assume that a social planner observes the joint distributions over $(v, c)$ and can arbitrarily set prices, but-consistent with the notion of asymmetric information-does not observe $c_{i}$ for any

\footnotetext{
${ }^{14}$ In other words, although industry marginal cost curve may have an arbitrary shape, there is no association between the cost of insuring a particular consumer and her valuation of the contract.

${ }^{15}$ In this paper, the focus is on the social planner's problem of optimal pricing in order to highlight the phenomenon of interest. Nonetheless, the competitive equilibrium without regulator intervention can be seen in Figure 1 at the intersection of the implied average cost curve and demand.
} 
specific individual, and therefore cannot price as a function of unobservable consumer costs. It is nonetheless true that in each of the panels in Figure 1, it is possible to draw a single price that sorts all consumers efficiently. Horizontal lines at $p^{*}$ in the figure show the efficient price.

Now consider Figure 2, which introduces the feature of interest: selection interacting with demand heterogeneity. For each level of costs along the horizontal axis in Panel A, there existent two different valuations. In this case there is no price that can induce efficient sorting. To see this, note the candidate price $p$ plotted as a dashed horizontal line. Prices must be lower than $p$ to sort type $x$ efficiently. But it must be higher than $p$ to sort type $y$ efficiently. Clearly, no single price can satisfy these criteria simultaneously. The allocative efficiency problem can also be seen in the corresponding demand diagram in panel B of Figure 2. No horizontal price line can be drawn such that $v_{i} \geq p$ if and only if $v_{i} \geq c_{i}{ }^{16}$

In the presence of demand heterogeneity, marginal costs no longer trace a single curve but rather become a cloud of points. Because there is no unique intersection point between this cloud and the demand curve, there is no uniform price that generates efficient consumption.

The general point about the inefficiency of price as a sorting mechanism for selection markets with demand heterogeneity has been briefly noted by Einav and Finkelstein (2011) and explored in more detail by Bundorf, Levin and Mahoney (2012) and Glazer and McGuire (2011). Panel A of Figure 2, in fact, closely parallels a plot in Bundorf, Levin and Mahoney (2012). ${ }^{17}$ However, with the exception of Glazer and McGuire (2011), the literature has universally focused on cost-based pricing or on a second-best response of the planner who sets the best possible uniform price, like $p$ in Figure 2. In the next section, I show that there may be a feasible improvement relative to the best uniform price. Developing this finding theoretically and then demonstrating that such cases may be empirically relevant are the main goals of this paper.

\subsection{Price Discrimination on Preferences}

A feasible, welfare-improving refinement relative to the best uniform price may be possible if there exists an observable correlate of demand. Feasible here means that costs remain unobservable and cannot be used in pricing, consistent with the notion of asymmetric information. I begin with graph-

\footnotetext{
${ }^{16}$ Although $v$ and $c$ are positively correlated overall in Figure 2, the relationship is not monotonic, and there are points $i$ and $i^{\prime}$ for which $v_{i}>v_{i^{\prime}}$ even though $c_{i} \leq c_{i^{\prime}}$.

${ }^{17}$ This possibility of a marginal cost cloud is also conjectured in Einav, Finkelstein and Cullen (2010), who comment in a footnote: "Note that there could be multiple marginal consumers."
} 
ical intuition and then formalize the finding.

Panel A of Figure 3 repeats Panel A of Figure 2 with one difference: The scatter points representing individuals are now identifiable as belonging to two observable groups. Group $a$ is represented by hollow circles, group $b$ by solid circles. The identifiable types could represent income, wealth, sex, age, or any other observable. It is important to note, however, that by construction, the two groups exhibit identical risk distributions (i.e., the distribution of the circles along the horizontal axis). This construction isolates the phenomenon of interest, which is heterogeneity in preferences or demand, not heterogeneity in costs. The consumer groups $a$ and $b$ differ only in willingness-to-pay for insurance.

In the case of Figure 3, it is straightforward to see that a welfare-improving policy is to segment the market by preference type, setting different prices for groups $a$ and $b .{ }^{18}$ The prices $p_{a}^{*}$ and $p_{b}^{*}$ that sort all consumers efficiently are depicted in the figure. In Panel B, two separate demand diagrams for the $a$ and $b$ markets are overlaid. The overlay highlights that selection within the $a$ and $b$ markets produces exactly the same marginal cost curves, but differences in $v$ conditional on $c$ generate different demand and therefore different optimal pricing. Despite the fact that the joint distribution of valuations and costs are exactly the same as in Panel A of Figure 2, the market segmentation allows prices to efficiently sort consumers. Panel $\mathrm{C}$ shows the result for an analogous case with a large number of consumers in each group and continuous distributions of costs.

To formalize the intuition of Figure 3, consider an observable characteristic $z$ that is contained in consumer charactistics $\psi$ and/or $\delta$ and is therefore correlated with willingness-to-pay. If $z$ remains correlated with willingness-to-pay after conditioning on expected costs, then optimal pricing within segments partitioned along the characteristic $z$ is welfare improving relative to the optimal uniform price. The proof is developed in Appendix A.1. It follows the same intuition of the diagram in Figure 3: Prices that induce efficient allocations are set where the demand and cost curves cross, and since these curves have different crossing points among groups for which $v$ systematically differs conditional on $c$, segmenting the market by the characteristic $z$ raises total surplus.

The novel idea of the model is that price discrimination across groups with systematically different preferences is optimal. This is not because these groups face different insurable risk (which

\footnotetext{
${ }^{18}$ Here, "preference" is merely shorthand for demand heterogeneity that is conditional on costs. The model captures a broad notion of heterogeneity that includes differences in preferences, differences in constraints, and differences in higher moments of the risk distribution, any of which could affect demand conditional on expected insurer costs.
} 
by construction they do not) but rather because these groups value insurance contracts differently conditional on facing the same risk. This is the key insight of the model that is distinct from other work that has considered segmenting selection markets according to cost types.

For completeness, Appendix Figure A1 graphs the case that corresponds most closely to the notion that young and old, rich and poor, men and women, etc., would differ in insurance demand only because of cost differences. It is straightforward to observe in Figure A1 that under this assumption, costs perfectly align with valuations, and therefore even if types could be identified and the market segmented, there would be no welfare gain to price discrimination, relative to the constrained optimal uniform price.

\subsection{A Sufficient Statistic Test}

The last section discussed consumer valuations of insurance contracts, but the econometrician observes insurance choices, not underlying valuations. The natural analogue to the partial correlation between observables and plan valuations $\left(\rho_{v, z \mid c}\right)$ is the partial correlation between observables and plan choices $\left(\rho_{I(j), z \mid c}\right)$.

Let $I(z=a)$ and $I(z=b)$ represent indicator functions denoting membership in groups $a$ and $b$, and let $I(j)$ indicate enrollment in plan $j$. By revealed preference, satisfying the inequality with respect to plan choices

$$
E[I(j) \mid c, I(z=a)] \neq E[I(j) \mid c, I(z=b)]
$$

is a sufficient condition for identifying differences in plan valuations between types $a$ and $b .{ }^{19}$ If the condition in (5) is met and if there is selection in the market, then price discrimination on $z$ yields a feasible improvement relative to the best uniform price. As I illustrate in the next section, checking the condition in Expression (5) is simple with the kind of information readily-available in health claims data.

Expression (5) is is not a test for selection. Although (5) parallels the widely applied Chiappori and Salanie (2000) test for selection in insurance markets in terms of form and simplicity, it answers a very different question. Abstracting from moral hazard, the Chiappori and Salanie test and its many implementations (e.g., Finkelstein and Poterba, 2004) is a test for positive correlation between

\footnotetext{
${ }^{19}$ Differences in enrollment imply differences in valuations (though the converse is not necessarily true): If valuations were identical, and because prices are identical by construction, choices would have to be identical. Therefore, $\rho_{I(j), z \mid c} \neq 0$ implies $\rho_{v, z \mid c} \neq 0$.
} 
purchasing a more generous contract and relevant loss-related outcomes, such as the claims costs born by insurers. It takes a form like $E[c \mid I(j)] \neq E[c \mid I(k)]$ and asks whether there is selection on costs across plans. The test in (5) indicates nothing about selection on costs. Instead, it asks whether one group of consumers reveals different preferences over plans, holding the cost they generate for the insurer fixed.

Implementing the test in (5) requires generating an estimate, $\widehat{c_{i}}$, of each individual's expected costs, $c_{i}$. Because most observable consumer characteristics (e.g., age, sex, income, wealth) will be correlated both with contract valuations and also with expected costs, it is important to demonstrate empirically that the control $\widehat{c_{i}}$ is sufficient to remove any partial correlation between $z_{i}$ and $c_{i}$. I discuss the practical issues of estimation in detail in Section 3.3 after introducing the data.

\subsection{Incorporating More Complex Heterogeneity}

The case in Figure 3 is a conceptually useful starting point, but two complications are likely to be relevant in real-world settings, including in the empirical setting of this paper. First, identifiable groups will likely differ from each other not only in valuations but also in risk profiles. Second, residual demand heterogeneity will always exist within whatever groups can be identified for segmentation. For example, as I show below in Section 6, women have greater demand for insurance than men, holding expected losses fixed, but within the male and female groups there remains substantial idiosyncratic heterogeneity in revealed preference. It is essentially this within-group heterogeneity that Bundorf, Levin and Mahoney (2012) study.

Figure 4 incorporates cost differences across groups and within-group demand dispersion while maintaining the feature of Figure 3 that, on average, there are differences in demand across the two groups. The panels parallel Panels A and B of Figure 3, but here valuations across individuals within the $a / b$ types also differ conditional on cost, or equivalently, costs differ conditional on valuation. As above, individuals are represented by points. The top panel is plotted in $(v, c)$ space and includes a 45-degree line that separates efficient from inefficient enrollment. The bottom panel plots the corresponding demand diagram.

As in Figure 3, these assumptions generate different optimal prices for the two types, $p_{a}^{*}$ and $p_{b}^{*}$. In contrast, now the group with the higher willingness-to-pay conditional on cost, type $a$, optimally faces higher prices. Note that costs overlap across groups at exactly one point, where the costs of indi- 
viduals $x$ (type $b$ ) and $y$ (type $a$ ) are identical. Conditional on this common cost, types $a$ have higher willingness-to-pay. Thus, allowing for within-group demand dispersion is theoretically important. In particular, it may no longer be the case that the group with higher demand optimally faces lower prices.

Within-group heterogeneity rules out the possibility of a first-best allocation. It nonetheless remains true that price discrimination across the $a / b$ types is constrained optimal relative to the best non-discriminatory price. In the example of Figure 4, price discrimination leads to more individuals self-sorting efficiently, even if no prices can sort all consumers efficiently.

\subsection{Interaction with Risk Adjustment}

The textbook solution to Akerlof-style price distortions is consumer subsidies that have the effect of lowering the uniform price that consumers face. Another prominent regulatory response to selection in health insurance markets is risk adjustment. ${ }^{20}$ Risk adjustment works as a non-uniform subsidy to insurers that varies with consumers' expected cost types, $c_{i}$. It compensates insurers or plans that enroll low expected cost types and taxes insurers or plans that enroll high expected cost types. In terms of the demand diagram in Figure 3, risk adjustment flattens the insurer's perceived marginal cost curve, net of the risk-adjusted transfers. (Social efficiency nonetheless occurs along the actual marginal cost curve.) With the proper level of risk adjustment subsidies, the regulator could implement any uniform price as a competitive market equilibrium outcome, just as with direct consumer subsidies. $^{21}$

However, risk adjustment does nothing to address the inefficiency arising from non-discriminatory prices in a setting with demand heterogeneity. Risk adjustment does not alter the facts that (i) consumers with different demand face the same prices, and (ii) consumers with different demand must face different prices to sort themselves efficiently. Research subsequent to this study, including Layton (2014) and Layton, Ellis and McGuire (2015), has examined this interplay of risk adjustment and demand heterogeneity.

\footnotetext{
${ }^{20}$ See Van de Ven and Ellis (2000) and Breyer et al. (2011) for overviews.

${ }^{21}$ The compensation $\left(R A_{i}\right)$ is typically equal to the difference between expected costs as predicted by the regulator $\left(\widehat{c}_{i}\right)$ and mean costs in the population $(\bar{c}): R A_{i}=\widehat{c_{i}}-\bar{c}$. Perfectly functioning risk adjustment can flatten the firm's perceived cost curve. Optimal uniform pricing requires an additional refinement: a lump-sum subsidy that serves to set the level of the firm's perceived constant average cost at the level where demand and unadjusted marginal cost would otherwise intercept. Risk-adjusted payments to achieve the constrained optimum under uniform pricing would take the form $R A_{i}^{*}=$ $\widehat{c_{i}}-\bar{c}+\eta$, where $\eta$ is this appropriate lump-sum subsidy, which could be financed through a flat tax across all plans.
} 


\section{Data and Reduced Form Empirical Strategy}

\subsection{Data}

I examine consumer plan choices in an employer-sponsored health insurance setting to demonstrate that the kind of demand heterogeneity described in Section 2 is empirically relevant. The employer health plan setting is uniquely well suited for identifying the effects of interest for several reasons. First-unlike other settings, such as the ACA Marketplaces or other individual markets-one can observe men and women, young and old, sick and healthy, rich and poor, all facing the same menu of insurance options at the same prices. Second, the particular plans here are differentiated only in cost sharing-not provider networks or other plan features. This facilitates a straightforward comparison of the plan options in an expected utility framework, which I exploit in Section 5. Finally, the detailed claims data contains all the information needed to calculate unbiased estimates of the insurer's marginal costs.

Data come from the administrative health insurance records from a large anonymous employer. ${ }^{22}$ Employees in the firm were offered the choice of two vertically differentiated Preferred Provider Organization (PPO) plan options. These plans differed only in their cost-sharing rules—that is, the degree of consumption smoothing they provided. Denote the two contract options $L$ and $H$ for low and high coverage. Compared to $L$, contract $H$ had a lower deductible ( $\$ 300$ versus $\$ 500$ ), lower coinsurance $(10 \%$ versus $20 \%)$, and a lower out-of-pocket maximum $(\$ 2,300$ versus $\$ 4,250) .{ }^{23}$ Table 1 summarizes the plans.

The employee contributions to plan premiums are not available in the data, so the prices of these plans are not known. In contrast, average plan costs, which are systematically related to premiums in competitive market settings as well as in most employer-subsidized plan settings, can be directly and precisely calculated by aggregating the total claims paid out by each plan. ${ }^{24}$ Fortunately, identifying demand heterogeneity across groups (the test of Equation 5) only requires observing the plan choices of consumers who face the same options at the same prices. It does not require knowing what those

\footnotetext{
${ }^{22}$ The source is the Medstat Commercial Claims Database, accessed via the NBER.

${ }^{23}$ The coinsurance rate is the marginal price faced by the consumer once the deductible has been met. Both the deductible and coinsurance are counted towards the out-of-pocket maximum. The cost sharing rules in Table 1 apply only to innetwork services. Out-of-network services, which comprise a small minority of all claims, involve higher cost sharing in both plans.

${ }^{24}$ When prices are needed in the structural choice model below, they are constructed by applying typical employee contribution formulas to average plan costs.
} 
prices are.

The data are composed of service-level claims files and employee enrollment records. Claims include the line-item billing and diagnosis information on all enrollees. This covers every contact with a medical service provider and all prescription drug purchases. Besides prices and quantities, claims records list the portion of each bill paid by the enrollee and by the health plan. Most records include one or more diagnosis or procedure codes. The demographic and diagnoses information on patients $(\delta)$ combined with plan cost-sharing rules $(\phi)$ are used to construct a measure of expected insurer payouts that corresponds to the term $\widehat{c(\phi, \delta)}$ in the model of Section 2.

The main estimation sample is limited to the employees who enroll themselves without dependents in one of the employer's plans. ${ }^{25}$ The claims data span 2004 through 2007. To avoid the complication of enrollees aging into Medicare and out of the sample over the panel, I cap the sample at age 59. This results in a sample of about 22,000 enrollee-years, composed of $60 \%$ males, with an approximately uniform age distribution between 18 and 59. Appendix Section A.2 gives additional details on worker and firm characteristics.

Figure 5 shows the detailed distribution of healthcare spending in the sample by age and sex. The figure plots a kernel density estimate of positive spending and separately indicates the fraction in each subsample without any healthcare utilization. Utilization increases with age and is higher for women than men. The figure shows that within these demographic groups and overall, healthcare spending in the sample follows a mixture distribution that includes a point mass at zero and an approximately log-normal component.

Table 2 provides a high-level summary of employees' plan choices and healthcare spending. The first column tallies the fraction of enrollees who chose the more generous insurance option, plan $H$. Healthcare consumption in columns (2) through (4) is measured as the total bill paid to service providers. Column (2) gives the average overall healthcare expenditure. Columns (3) and (4) display these average healthcare costs conditional on the plan chosen. The first row lists these aggregate statistics for the entire estimation sample, pooling years 2005-2007. Consistent with most studies of selection in health insurance markets, adverse selection is reflected in the plan choices. Enrollees that

\footnotetext{
${ }^{25}$ Singly enrolled employees are the simplest group in which to draw a clean comparison of demand differences conditional on age and sex. Enrolled families, in contrast, are mixed demographic groups but must make a common health plan choice. Roughly one third of enrolled employees enrolled only themselves rather than themselves plus one or more family members. These employees may nonetheless have spouses and children who receive insurance through other channels, such as the spouse's employer.
} 
choose plan $H$ consume twice as much healthcare on average as plan $L$ enrollees. ${ }^{26}$ In the absence of preference heterogeneity, the inefficiency generated by this type of selection can be counteracted: with subsidies in a competitive market setting, or with properly set uniform premium subsidies in employer plans. Moving down the table, the same general pattern of adverse selection exists within each group as it does in the sample overall. The average level of healthcare costs varies widely across the groups. For instance, the average consumption of a 50- to 59-year-old is around 3.5 times that of an 18- to 29-year-old ( $\$ 6,305$ versus $\$ 1,820)$.

Importantly, Table 2 shows the first suggestive evidence that there could be significant demand heterogeneity within and across groups. A one-to-one mapping from insurable risk to plan takeup would tend to produce expected costs in plan $H$ that are higher than expected costs in plan $L$, overall and when comparing these costs across the age groups. In contrast, Table 2 shows that 50- to 59year-olds who choose the low coverage plan generate average costs of about $\$ 5,000$, while 18 - to 29 year-olds who choose the high coverage plan generate average costs of only $\$ 3,000$. This suggests that consumers are not uniformly following a common decision rule for when to takeup $H$. The summary statistics are merely suggestive on this point. The systematic analysis below compares plan choices between young and old enrollees who face the same ex ante level of expected healthcare costs.

\subsection{Regression Framework and Identification}

Abbreviating the indicator function notation (from $I(z=a)$ to $I_{a}$ ) and rearranging terms in (5) yields $E\left[E\left[I_{j} \mid I_{a}\right]-E\left[I_{j} \mid I_{b}\right] \mid c\right]$. This expression for the difference in the conditional expectations naturally maps to a regression framework. The expression is equal to the coefficient on the type indicator $\left(I_{z}\right)$ from an an OLS regression of plan choice on types $(z)$ that controls for costs $(c) .{ }^{27}$ Thus, the sufficient statistic test in (5) is reduced to examining coefficients on group indicators. Using the claims data, I

\footnotetext{
${ }^{26}$ Some fraction of the spending differences across plans is likely due to moral hazard. Given the common range of estimates for the elasticity of healthcare consumption with respect to out-of-pocket price, this would be a small fraction. For example, the RAND HIE (Manning et al., 1987) estimates this elasticity to be -0.2 . Applying this parameter and the $10 \%$ coinsurance difference between $L$ and $H$ to the average health spending in $L$ would imply that the counterfactual spending of plan $L$ enrollees if they were observed instead in plan $H$ would be $\$ 3,028(=\$ 2,672+\$ 2,672 \times(-0.10 / .15) \times(-0.2))$. Moral hazard would thus explain little of the observed spending difference between plans $H(\$ 6,090)$ and $L(\$ 2,672)$.

${ }^{27}$ See Angrist and Pischke (2008), Chapter 3. If the conditional expectation functions are linear, then the OLS regression $I_{j_{i}}=\sum_{z \in Z} B_{z} I_{z_{i}}+\beta_{c} c_{i}+\epsilon_{i}$ is just the conditional expectation. Otherwise, the OLS regression gives the best linear predictor of $I_{j}$ given $(c, z)$.
} 
implement the test by estimating OLS regressions of the form:

$$
H_{i}=\sum_{z \in Z} \beta_{z} I_{z_{i}}+f\left(c_{i}\right)+\beta_{X} X_{i}+\epsilon_{i}
$$

where $H_{i}$ is an indicator for choosing plan $H$, and $I_{z}$ are indicators for sex and age bins. The coefficients of interest are $\beta_{z}$. The cost variable $c_{i}$ is described more below, and in various specifications is controlled for linearly or non-parametrically. To maximize power, individuals observed in several years of data are included, with standard errors clustered at the person level. Year effects are included in all regressions. Additional controls $X$ are described below.

Irrespective of the control set included, the coefficients of interest, $\beta_{z}$, are not interpreted as causal effects. Several unobservables that are presumably important in the consumer's decision process (e.g., risk aversion, wealth at risk, the dispersion of risk) would drive correlation between $I_{z_{i}}$ and $\epsilon_{i}$. This poses no problem for the identification strategy. Any correlation between an observable $z$ and demand is evidence of the phenomenon described by the model in Section 2. This is precisely because it indicates that $z$ is a correlate of some unobserved preference or constraint that is generating demand differences.

In this sense, the regression in Equation (6) constitutes a sufficient statistic test: It reveals demand differences without making claims about the underlying primitives that generate the demand. Any utility function primitives or constraints that would generate the same demand curves would have the same implications for welfare. ${ }^{28}$

With that in mind, the only appropriate controls $X$ to include in the regression would be those that could impact contract choice through some channel other than via correlation with the enrollee's insurance demand. One potential confounder of this type would be if the regression failed to adequately limit the sample to employees facing the same choice set and premiums. Focusing on the employer setting - and in particular, focusing on a single firm offering a common choice set to all enrollees-essentially eliminates the possibility of this type of misspecification. The reason is that

\footnotetext{
${ }^{28}$ In other words, the point of view in the regression is that of a social planner or risk-neutral insurer who is concerned with plan choice as a function of cost to insure, but not necessarily with the preferences or budget constraints that generate demand. Einav, Finkelstein and Cullen (2010) summarize this point about sufficient statistics in welfare analysis in the following way: "Different underlying primitives (i.e. preferences and private information...) have the same welfare implications if they generate the same demand and cost curves." The only set of explanations for which this fails to hold is if consumers have behavioral biases or incorrect subjective risk assessments. In that case, there is no clear correspondence between revealed preference and social welfare, and in general there is no widely accepted solution to welfare analyses in such cases.
} 
the Employee Retirement Income Security Act of 1974 (ERISA) and the Health Insurance Portability and Accountability Act of 1996 (HIPAA) prohibit employers from charging different premium contributions to employees of different gender, race, age, national origin, religion, or disability. ERISA requires that "similarly situated" employees face identical premium contributions. The only theoretically relevant controls are those that might enter into defining workers as "similarly situated." Therefore, I include indicators for hourly versus salary pay, full-time versus part-time employment, and union status. In practice, adding these controls has essentially zero impact on point estimates and confidence intervals.

\subsection{Expected Cost Measure}

To control for expected costs, I use the Johns Hopkins Adjusted Clinical Grouper (ACG) to aggregate the rich clinical information contained in the claims files into a predicted expected health spending measure $\widehat{C}_{i}$. The algorithm predicts period $t$ total spending at the individual level using demographics and $t-1$ diagnosis and utilization information. ${ }^{29}$ This approach and tool has been used and validated in the recent literature, including in Handel (2013) and Handel, Kolstad and Spinnewijn (2015). The resulting prediction is for total healthcare consumption in dollars-that is, the total (enrollee plus insurer) payments made to providers. For various parts of the analysis below, I convert health predicted health spending, $\widehat{C}_{i}$, to the insurer's predicted cost, $\widehat{c_{i j}}$, by applying plan cost-sharing rules to the total spending amount.

For the sufficient statistic test embodied in Equation (6), $\widehat{c_{i}}$ need only satisfy two conditions. First it must be an unbiased predictor of insurer costs. Second, controlling for $\widehat{c_{i}}$ must eliminate any correlation between the observable type and expected costs: $\rho_{c, z \mid \widehat{c}}=0$. The panel nature of the data makes it possible to directly test these conditions by comparing the prediction of expected costs to the ex-post realized claims costs. In Section 4, I confirm that a regression of realized costs on predicted costs yields a slope coefficient near one and an unconstrained intercept of zero. ${ }^{30}$

\footnotetext{
${ }^{29}$ Inputs into the ACG from my data include age, sex, pharmacy spending, medical (non-pharmacy) spending, inpatient stays, outpatient visits, ER visits, all ICD-10 diagnosis codes, all CPT procedure codes, national drug codes of all prescriptions filled, and places of service.

${ }^{30}$ Alternative expected cost measures, including the predicted values from my own regressions of current expenditure on past expenditure, run separately by age and sex, produce similar results to those shown in the next section. Unsurprisingly, less of the variance in realized healthcare costs is explained by these alternative predictors.
} 


\section{Results}

In this section, I present the main empirical finding of the paper: that willingness-to-pay for insurance covaries strongly with age and sex, after fulling accounting for expected (or realized) healthcare spending differences. Here, the intention is to document patterns of demand that match the model, while imposing minimal economic and econometric assumptions. In Section 6, I test additional predictions of the model and calculate policy counterfactuals by assuming an expected utility framework that imposes more restrictive assumptions.

\subsection{Main Results: Demand Patterns Across Age and Sex}

Figure 6 plots local polynomial regressions of plan choice on expected costs estimated separately by age group in the top panels and by sex in the bottom panels. These plots are semi-parametric versions of the linear regression in Equation (6). The dependent variable is an indicator for choosing plan $H$, which is approximately linear in log expected costs. In Panels A and C (left), I use total expected

healthcare spending, $\widehat{C}_{i}$, as the cost control variable. This specification illustrates the relationship between insurance demand and medical risk with minimal data manipulation. In Panels B and D (right), I condition instead on the theoretically relevant measure from Equation (5): the difference in expected insurer spending between the plans that is implied by the individual's total expected healthcare spending, $\widehat{c}_{i \mathrm{H}}-\widehat{c}_{i \mathrm{~L}}$.

There is a natural parallel between the empirical results in Figure 6 and the model of Figure 2. Figure 6 plots plan takeup and Panel A of Figure 3 plots plan valuations. The takeup rate is just the fraction of consumers for whom willingness-to-pay for plan $H$ over plan $L\left(v_{i H}-v_{i L}\right)$ exceeds the difference in employee premium contributions between these plans. The plots show that conditional on either measure of expected healthcare costs, takeup of $H$ increases monotonically in age. Women also appear to have a higher willingness-to-pay for $H$. To understand the size of the demand differences implied by the plots, consider holding takeup of plan $H$ fixed at $40 \%$ in the top left panel of Figure 6. Takeup rates reach $40 \%$ at approximately $\$ 2,000$ of expected spending for 50 - to 59 -year-olds. But among 18- to 29 -year-olds, takeup rates reach $40 \%$ only at $\$ 8,000$ of expected spending.

To assess statistical significance, as well as control for other covariates, Table 3 reports results from a series of plan choice regressions. Guided by the log-linearity apparent in Figure 6, I regress an indicator for choosing plan $H$ on the natural logs of expected costs. Columns (1) through (3) 
evaluate age and sex coefficients in the same regression and add controls for state fixed effects and worker characteristics. These controls are important in principle if workers at different plant locations or with different status (hourly/salary, part-time/full-time, unionized/not) face different premium contributions. In practice, the controls have almost no impact on coefficients or significance. Columns (4) and (5) evaluate age and sex separately—a relevant separation for considering markets in which regulators allow pricing on age but not sex, or on sex but not age.

Consistent with the semi-parametric plots, the regressions in Table 3 confirm there is significant demand heterogeneity that is associated with observables. Coefficients on the age dummies can be interpreted as percentage point differences in takeup rates relative to the excluded category, 18- to 29-year-olds. These estimated coefficients correspond to large differences. Taking parameters from column (3), mean takeup rates among 18 - to 29 -year-olds are $42 \%$ lower $(=.093 / .220)$ than among 50 to 59 year-olds who are equally costly to insure. Differences across sex are less pronounced than across age, but the coefficients indicate that women have a stronger preference on average for more comprehensive insurance. The fact that demographic groups differ not only in underlying risk but also in insurance demand conditional on risk is a pattern necessarily missed by studies that have, in the absence of detailed claims data, relied on demographic variables as proxies for health expenditure risk.

It is important to note that age and sex are not assumed to be exogenous in these regressions. These observables are merely correlated with a variety of unobserved parameters and constraints that are contained in the error term. As the analysis in Section 2 demonstrated, this correlation between an observable characteristic and unobserved demand determinants is exactly the pattern of interest because it indicates that such observables can be used by the social planner or regulator to more efficiently price discriminate. To the extent that the age and sex patterns shown here carry over to other markets like Medicare, Medicaid, and the ACA Marketplaces, these results are informative of the potential inefficiency of non-discriminatory pricing regulations in these other markets.

\subsection{Robustness}

Although age and sex need not be uncorrelated with the error term in Equation (6), identification nonetheless requires that these observables have no residual correlation with insurer losses after controlling for $\widehat{C_{i t}}$. If there were bias in the expected costs measure that differed systematically by 
age, this would pose an identification problem. ${ }^{31}$ The panel nature of the data permits a direct test for this type of bias because the prediction $\widehat{C_{i t}}$, which is based on diagnosis and utilization information from the preceding plan year $(t-1)$, can be compared to its realized value, $C_{i t}$. It is straightforward to test whether age remains correlated with realized spending after conditioning on $\widehat{C_{i t}}$.

Table 4 displays the results from a series of regressions of realized healthcare spending on the expected spending measure, with and without controls for age. ${ }^{32}$ As a baseline, the regression in column (1) includes all enrollees in the main estimation sample, and $\widehat{C_{i t}}$ is the only regressor. Consistent with unbiased prediction, the slope is very close to one, and the intercept is close to zero. Columns (2) through (6) of Table 4 address the question of whether $\widehat{C_{i t}}$ is differentially biased by age. Column (2) adds age bin indicators to the regression. The F-statistic on the additional age variables demonstrates that these have no residual power $(p=0.72)$ to predict realized health spending after conditioning on $\widehat{C_{i t}}{ }^{33}$ Columns (3) through (6) rerun the regression separately by age group. Even in these more flexible specifications, there is no evidence of bias. For each of the subsamples comprising columns (3) through (6), the slope estimates are close to one, and the intercepts are economically small and not statistically different from zero. ${ }^{34}$ For completeness, Appendix Figure A2 plots a raw scatter of $C_{i t}$ versus $\widehat{C_{i t}}$ separately for each age bin.

Table 4 and Figure A2 provide clear evidence against the possibility that the observed differential demand patterns are spuriously driven by residual correlation between age and plan choice that is not partialed out by controlling for $\widehat{C_{i t}}$. It is important to note that even if-counter to the findings of Table 4 -there were some differential prediction error in $\widehat{C_{i t}}$ across age groups, it would be unlikely to be large enough to spuriously generate the magnitude of the observed demand differences. For example, Figure 6 shows that expected spending estimates would have to be biased by around $\$ 6,000$ between the youngest and oldest groups (about 400\% of the 18- to 29 -year old mean) in order for

\footnotetext{
${ }^{31}$ For example, the Johns Hopkins ACG tool could over-predict expected spending for the young and under-predict it for the old. If so, it would have the effect in Figure 6 of (spuriously) spreading the regression lines away from each other. A more subtle problem would occur if the ACG prediction had no differential bias across groups but suffered from measurement error. In that case, estimates of the age dummies would be biased as well, in the sense of no longer representing age effects that are independent of expected costs. For example, in the extreme, if the health risk variable were purely noise, one would expect large age coefficients, even if the true age effects were zero, because the age coefficients would simply capture the correlation between age and average risk. The test in Table 4 rules out this alternative hypothesis as what is generating the results.

${ }^{32}$ The regression is $C_{i t}=\alpha+\eta \widehat{C_{i t}}+\sum \beta_{a} I_{a}+\mu_{i t}$, which should yield a slope $\eta$ of one and an unconstrained intercept $\alpha$ equal to zero. Most importantly, controls for age group indicators $I_{a}$ should yield small and insignificant coefficient estimates.

${ }^{33}$ It is unsurprising that age has no residual predictive power in this regression, given that age is already included as an input to the ACG prediction of $\widehat{C_{i t}}$.

${ }^{34}$ The p-values on the difference between the estimated slope and 1.00 are never smaller than 0.20 .
} 
unbiased versions of the two nonparametric plots to overlap. If this level of bias were present, one would expect a coefficient on the age 50 to 59 indicator in column (2) of around this magnitude. In contrast, column (2) shows a precisely estimated zero for this coefficient.

It is also worth noting that moral hazard, or selection on moral hazard (Einav et al., 2013), is unlikely to account for the patterns of results in Table 3 and Figure 6. This is because the conditioning variable, $\widehat{C_{i t}}$, is based on $t-1$ information (primarily demographics and diagnoses) rather than the period $t$ realization of spending that occurs under the chosen plan's cost-sharing rules. However, if one assumed that moral hazard were a source of bias, it would bias against the observed results: Moral hazard would suggest that the unobserved plan $L$ spending among plan $H$ enrollees is overstated, as cost sharing is lower in $H$ than $L .^{35}$ Because the probability of choosing $H$ increases in age, this would imply that, on average, expected costs are overstated for older consumers relative to younger consumers. In the context of Figure 6, accounting for this would require differentially shifting the curves for the older groups leftward. However, this would have the effect of widening the observed gaps. Thus, if the measure of expected costs were contaminated by moral hazard, it would bias against the main empirical finding.

\subsection{What Drives Demand Differences?}

Uncovering the determinants of the choice patterns revealed in Table 3 is not a main goal of this paper. Nonetheless, the empirical results above prompt the question of why older consumers place such a high valuation on more complete insurance coverage. Is it preference heterogeneity? Differences in higher moments of the risk distribution? Unobserved (to the econometrician) private information? In this section, I take a first step to investigating this issue. However, it is important to note that under the standard neoclassical assumption that consumer choices reveal consumer preferences, these documented demand differences themselves are sufficient to indicate welfare-improving price discrimination by age. Any differences in utility parameters (e.g., risk attitudes) or in constraints (e.g., wealth) that would generate the same demand differences across groups would have the same welfare implications.

One conceptually straightforward possibility is that, conditional on expected spending, the variance of risk distributions is wider for older enrollees. Mean-preserving risk spreads would lead to

\footnotetext{
${ }^{35}$ See Footnote 26 for an illustrative calculation that considers counterfactual plan $H$ spending of plan $L$ enrollees, accounting for moral hazard.
} 
different plan choices, even holding fixed both risk preferences and expected spending. Table 5 investigates the possibility, beginning in column (1), by repeating the main result from Table 3 for ease of comparison. The next two columns test the hypothesis that the spread of risk, operationalized as prediction error conditional on the expectation, drives the results. Column (2) controls for the absolute value of the difference between expected and realized costs, $\left|C_{i t}-\widehat{C_{i t}}\right|$, and column (3) controls for its square. While, consistent with risk aversion, these variables are significant predictors of choosing $\mathrm{H}$, their inclusion in the regression does not alter the demand patterns by age group relative to column (1). Demand heterogeneity also persists in the structural model of Section 5 below, where differences in higher moments of risk by age are estimated and fully accounted for.

In Columns (4) and (5) of Table 5 and in the top panel of Appendix Figure A3, I examine whether differences in private information on health risk can account for the patterns in the data. The conditioning cost variable in these regressions is the actual ex-post healthcare spending, $C_{i t}$. This spending variable is realized a year or more after the time at which the plan choice is made. ${ }^{36}$ If the demand differences were to dissappear with the inclusion of this control, it might indicate that older and younger consumers differed in takeup because they differed in their ability to forecast healthcare use. To match the specification in column (1) and the apparent log-linearity in Figure A3, I take natural logs of this spending measure. Unlike expected spending, realized annual spending is zero for some enrollees. I take two approaches to handling the zeros. In column (4), I translate the data by adding \$100 to the total annual claims for each enrollee. In column (5), I drop the zeros. Both of these ad-hoc adjustments yield the same result: Conditioning on realized costs, the differences by age persist. The top panel of Figure A3 plots the semi-parametric versions of these regressions, revealing the same patterns. These findings rule out the possibility that some age-specific selection on private information is driving the correlation between age and demand in the main specification.

Another class of explanations is behavioral/psychological. Such explanations could have importantly different welfare implications because these phenomena imply that the principle of revealed preference is invalid, and so standard welfare analysis would likewise be invalidated. The setting here is ideal for identifying demand differences but not for examining the possibility of behavioral phenomena behind them. Nonetheless, to the extent possible in these data, I evaluate the role of two behavioral explanations shown to be important to health plan choice in other work: incorrect

\footnotetext{
${ }^{36}$ Plan elections are made in fall of the year prior to the plan year.
} 
subjective assessment of healthcare needs (Abaluck and Gruber, 2011) and inertia (Handel, 2013).

There is no linked survey that reports consumers' self-evaluation of health risk, so a direct comparison of subjective beliefs with objective risks is not feasible. However, one potentially salient datapoint for consumers may be last period's spending, which will be an imperfect proxy for future spending. If younger and older consumers have differential behavioral biases toward relying on this type of backward-looking heuristic, it could drive the observed demand differences of column (1), which are conditional on unbiased expected spending. Columns (6) and (7) analyze the possibility, controlling for last period's realized costs rather than expected costs for the coming plan year. Zeros in the realized spending measure are transformed in column (6) and dropped in (7). The corresponding semi-parametric plots are included in the bottom panel of Appendix Figure A3. The monotonic pattern of demand by age persists, providing some evidence against the possibility that younger and older employees make similar choices conditional on a last-period decision heuristic. ${ }^{37}$

With respect to inertia, the young and old may be differentially active or passive in their annual plan choices, and over time this may lead to systematically different patterns of choice conditional on expected costs. It is also possible that younger and older workers, on average, began their firm tenure during a period when plan options or defaults were different. ${ }^{38}$ Some insight into the importance of inertia and defaults can be gleaned by restricting the analysis to a smaller sample of employees who are entering their first year of health plan enrollment during the data series. In the first year, switching costs are likely to be smaller, the default option will be the same across all enrollees, and younger and older employees are more likely to have the same experience with the firm's plans.

To investigate, I limit the sample to employees in the first year of enrollment in columns (8) and (9) of Table 5. Expected healthcare consumption cannot be calculated for-first year enrollees since they have no medical history to use as a basis for prediction. Therefore, I use ex-post realized consumption from the first year of enrollment as an imperfect control for expected costs. The coefficients show that the demand heterogeneity across age is present even at initial enrollment. The differential enrollment patterns are therefore not likely to be driven by older employees "aging in place," by

\footnotetext{
${ }^{37}$ It is not expected that the coefficients on age indicators will be invariant across the columns of Table 5. Only conditioning on expected costs, as in column (1), removes the mechanical correlation between age and the cost variable that theory predicts should drive plan choice. The coefficients on age indicators in columns (2) through (5) will reflect both the partial correlation between age and choice, holding expected costs fixed, and the part of variation in age that is correlated with the omitted expected cost variable.

${ }^{38}$ It is unknown whether a default option existed for the first year of health plan enrollment. It would be typical to default enrollees into the lower cost plan, $L$.
} 
older employees entering employment at a time of different default options, or by older employees having more experience with the employer's health plans.

In sum, the data suggest that the revealed demand differences are not driven by higher moments of expenditure risk, residual private information, backward-looking heuristics, or inertia. Other plausible explanations that are an avenue for future investigation include differences in risk preferences and income. Unfortunately, there is very little scope to differentiate between these alternatives with the data at hand: There are no independent (e.g., survey-based) measures of risk aversion in this administrative data. With respect to income, a CRRA model would suggest that older employees, whose income and wealth is unobserved but is likely higher, would have lower willingness-to-pay for insurance, deepening the puzzle of the revealed patterns. However, as Mahoney (2015) shows, bankruptcy laws create a floor on financial risk, which in turn affects demand for health insurance. Acknowledging that older workers may have greater value at risk due to bankruptcy protections could reverse the predictions of lower insurance demand for the wealthy without departing from a CRRA (or CARA) expected utility framework. Another possibility related to income is differentially high liquidity constraints for younger workers. An important avenue for future work will be to develop research designs that can disentangle the sources of the demand differences that this paper highlights.

\section{Demand Estimation}

Interpreting the reduced form results of Section 4 through the lens of the model of Section 2 implies that uniform pricing regulations would generate inefficient insurance allocations in this setting. Precise numeric statements about optimal prices and welfare losses require tracing out the demand and marginal cost curves. In this section I estimate a structural discrete choice model that can be used to trace these curves, with the important caveat that unlike the reduced form results above, identification here requires assuming a particular functional form of utility. 


\subsection{Discrete Choice Model}

Consider a consumer facing the discrete choice between the two insurance contracts offered by the employer. ${ }^{39}$ The expected utility generated by these two contracts differs because the contracts differ in prices (i.e., employee premium contributions) and because they differ in the out-of-pocket spending risk to which they expose the consumer. In the empirical context of this paper, the choice between plans is potentially well described as a choice over money lotteries: In this setting the plans are distinguished in cost-sharing parameters alone, not in provider networks or in other plan features.

Following the literature, I assume expected utility of the constant absolute risk aversion (CARA) form. ${ }^{40}$ CARA implies that utility over money lotteries is invariant to wealth levels, which is a useful assumption because wealth is not observed in these data. The CARA utility index is

$$
u\left(W_{i}-P_{j}-O O P_{i j}\right)=-\frac{1}{\gamma} e^{-\gamma \cdot\left(W_{i}-P_{j}-O O P_{i j}\right)}
$$

where $\gamma$ is the risk aversion parameter. The term in parentheses is the money outcome that agent $i$ faces in each state of the world, conditional on choosing plan $j \in L, H$. This term consists of initial wealth, $W$, minus the employee's contribution to the plan premium, $P$, and the consumer's out-ofpocket spending, $O O P$.

To the basic utility index, I add terms that allow the model to capture differences across age and sex in how plans are valued. Unobserved idiosyncratic tastes that shift the relative valuations of the two plans are represented by $\epsilon_{i H}$, which is a normally distributed, mean zero error term: $\epsilon_{i H} \sim$ $N\left(0, \sigma_{e_{H}}^{2}\right)$.

Adding these additional taste parameters and integrating Equation 7 over states of the world yields the expected utility in each plan, $V_{i j}$. The states of the world are defined by healthcare utilization, and the relevant risk distribution is the individual $\times$ plan-specific out-of-pocket spending risk $F_{i j}(O O P)$ :

$$
V_{i j} \equiv E\left[u_{i j}\right]=\int-\frac{1}{\gamma} e^{\gamma \cdot\left(P_{j}+O O P-\delta_{0} H_{i}-\sum_{a} \delta_{a} I_{a_{i}} \times H_{i}-\delta_{F} I_{F_{i}} \times H_{i}-\epsilon_{i H}\right)} d F_{i j}(O O P) .
$$

\footnotetext{
${ }^{39}$ Because employers typically contribute significantly to premiums, the relevant decision for most employees is which plan to purchase rather than the choice of gaining insurance coverage versus not. In the model, this is represented by the outside option being defined as choosing the lower coverage plan, $L$. The choice of remaining uninsured is not modeled, and employees choosing to remain uninsured are not observable in the data.

${ }^{40}$ CARA preferences are typically an explicit or implicit assumption in the literature. Assuming CARA allows direct comparison of my estimate of risk aversion with other studies. See Table 7 below.
} 
The vector $I_{a} \times H$ is a set of interactions between a plan $H$ indicator and indicators for the same four age groups defined in Section $4 . I_{F} \times H$ is an interaction between female and plan $H$. The $\delta$ coefficients on these interactions are the estimates of interest, analogous to the age and sex coefficients in the Table 3 regressions. $\delta_{a}$ and $\delta_{F}$ capture preferences for plan $H$ that differ systematically across demographic groups. $\delta_{o}$ allows for the possibility that all consumers over- or under-value plan $H$ in a way that cannot be rationalized by adjusting the risk aversion parameter. Because the $\delta$ coefficients enter the utility function in the money term, they measure plan preferences in dollars. The error term, as well as the age and sex coefficients, are normalized to zero for plan $L$ since only differences between the two plans are identified. Likewise, the wealth term $e^{-\gamma \cdot W_{i}}$ that appears on both sides of the inequality $V_{i L} \gtrless V_{i H}$ can be dropped. ${ }^{41}$

Consumers compare $V_{i L}$ to $V_{i H}$ and choose the plan that yields the highest expected utility, given their characteristics $\left(\delta_{a}, \delta_{F}\right)$, idiosyncratic preferences $\left(\epsilon_{H}\right)$, and the out-of-pocket sending risk they face under each plan $\left(F_{i j}(O O P)\right)$. The choice problem aligns closely with the general canonical model described by Equation (1).

\subsection{Estimation}

The full parameter vector to be estimated is $\left[\gamma, \delta_{0}, \delta_{a}, \delta_{F}, \sigma_{e_{H}}\right]$. An input to the estimation process is the person $\times$ plan out-of-pocket spending risk distributions. I construct $F_{i H}(O O P)$ and $F_{i L}(O O P)$ in two steps. First, I estimate person-specific distributions of medical utilization risk, $G_{i}(s)$. Then I map these to out-of-pocket spending by mechanically applying each plan's cost-sharing rules.

To generate $G_{i}(s)$, I begin by creating cells defined by deciles of expected spending separately within each age group. Following Handel (2013), enrollees in the same cell are assumed to face the same ex-ante distribution of healthcare risk. Under this assumption, the ex-post distribution of observed healthcare consumption within a cell identifies its ex-ante distribution. Following a typical parameterization and consistent with the kernel density plots in Figure 5, healthcare utilization is assumed to follow a mixture distribution consisting of a lognormal component $(\log (u) \sim N(\mu, \sigma))$

\footnotetext{
${ }^{41}$ Wealth, which is not observed empirically, plays no role in plan choice under CARA preferences. This is apparent in the inequality that determines the takeup of plan $H: V_{i H}>V_{i L}$, which is $\int-\frac{1}{\gamma} e^{-\gamma W_{i}}$. $e^{\gamma\left(P_{H}+O O P-\delta_{0} H-\sum_{a} \delta_{a} I_{a} \times H-\delta_{F} I_{F} \times H-\epsilon_{i H}\right)} d F_{i H}(O O P)>\int-\frac{1}{\gamma} e^{-\gamma W_{i}} \cdot e^{\gamma\left(P_{L}+O O P\right)} d F_{i L}(O O P)$. Here the wealth terms cancel. For the same reason, only the price difference between plans $L$ and $H$ is needed for estimation.
} 
and a point mass at zero of probability weight $g .{ }^{42}$ I estimate the parameters of the mixture distribution $(g, \mu, \sigma)$ separately for each cell via maximum likelihood and then assign these to each individual within the cell. Finally, I convert healthcare utilization at each point in the distribution $G_{i}$ into outof-pocket costs $F_{i j}$ by applying the cost-sharing rules (deductible, coinsurance, and out-of-pocket maximum) of each plan. ${ }^{43}$

Simulated maximum likelihood (SMLE) is used to estimate the parameter vector. ${ }^{44}$ The procedure, which closely tracks Handel (2013), is described in full detail in Appendix Section A.3. Intuitively, the process numerically integrates over the enrollee-specific healthcare spending distribution to calculate expected utility in each plan, choosing parameters $\gamma, \delta_{0}, \delta_{a}, \delta_{F}, \sigma_{e_{H}}$ that maximize the likelihood of generating the observed choice patterns.

\subsection{Identification}

It is important to understand that precision in statements about welfare comes at the cost of imposing the assumption of CARA utility. In particular, in the absence of supply-shock-driven premium variation to trace out demand curves, the expected utility assumption exploits instead the substantial variation across enrollees in the money lottery implied by each health plan. This variation arises from idiosyncratic health spending risk. In other words, the assumption of an expected utility decision process allows one to infer responses to plan prices (which do not vary in the data) by observing responses to other plan costs (which vary considerably across individuals in the data). This is a strong assumption, and for that reason, the simulations in Section 6 should be viewed as a back-ofthe-envelope exercise that extrapolates away from the transparently identified reduced-form results of Section $4 .^{45}$

In contrast, the identification of demand differences across age and sex in the estimation of (8) does not rely on the assumption of an expected utility decision process. Instead, demand differences

\footnotetext{
${ }^{42}$ Lognormal with a point mass at zero is a commonly used parameterization of medical risk. A mixture of a point mass with a two parameter Weibull—an alternative that is less widely used—fits these data less well. It underestimates the right tail of the distribution.

${ }^{43}$ To focus on the welfare implications of the demand heterogeneity of interest here, a maintained assumption is that healthcare consumption will be identical under either plan. This is equivalent to an assumption of no moral hazard. See footnote 26 for a back-of-the envelope calculation regarding the probable importance of moral hazard relative to selection in this setting.

${ }^{44}$ Simulation is needed to calculate likelihood values because there is no closed-form expression for the choice probabilities implied by Equation (8).

${ }^{45}$ For example, the expected utility assumption would be violated if, as a behavioral/physchological phenomenon, consumers viewed spending $\$ 50$ on a monthly premium differently from spending $\$ 50$ on a physician bill.
} 
across demographic groups continue to be identified by the institutional feature that all similarly situated employees face the same prices for the same insurance contracts. The coefficients on the $H \times$ $I_{a}$ and $H \times I_{F}$ interactions pick up effects that are not well described or rationalized by an expected utility framework that assumes agents within these different demographic groups have identical preferences and differ in demand only because of the differences in risk they face. ${ }^{46}$

Finally, plan costs are observed, but employer contributions to plan costs are not, which means that the price difference between contracts $L$ and $H$ that employees face is unknown. Following typical employer premium-setting patterns during this period, ${ }^{47}$ the price difference is assumed to be $20 \%$ of the employers' equilibrium average cost difference of providing plans $L$ and $H(=\$ 695) .{ }^{48}$ In estimation, this unobserved price enters identically to the plan $H$ intercept, $\delta_{0}$. Therefore, changing the assumed price difference changes the intercept coefficient dollar-for-dollar but affects no other coefficient estimates. ${ }^{49}$ Intuitively, the $\delta_{0}$ coefficient measures how, conditional on a risk aversion parameter and objective risk, all consumers over- or under-value contract $H$ relative to its price. As the assumed price changes, $\delta_{0}$ offsets it exactly.

\subsection{Parameter Estimates}

Table 6 lists estimated parameter values. Willingness-to-pay for plan $H$ increases monotonically in age. The coefficients indicate that, after controlling for objective risk, 30- to 39-year-olds value the fuller insurance option by $\$ 261$ more than 18- to 29 -year-olds. 40 - to 49 -year-olds value it by $\$ 323$ more. 50- to 59-year-olds value it by $\$ 508$ more. Willingness-to-pay is also somewhat higher among women. ${ }^{50}$ These patterns track the reduced form results of Section 4 . The estimated standard de-

\footnotetext{
${ }^{46}$ I also tried an alternative specification that allowed the risk aversion coefficient to vary by age, rather than including interactions of age and plan $H$ dummies in the money term. In practice, the model in which age entered via risk aversion did not fit the data well. This is likely because preference for $H$ within each age group was not correlated with dispersion in the OOP spending distribution, which such a specification would require. A separate benefit of the formulation in Equation (8) is that the estimates yield easily interpretable differences in valuation that are measured in dollars.

${ }^{47}$ For example, in 2007 for employee-only coverage in large firms, workers on average contributed 16\% of the premium, with firms contributing 84\% (Kaiser Family Foundation and the Health Research and Educational Trust, 2007).

${ }^{48}$ Starting from the average total spending in $L$ and $H$ from Table 2, the employer's share of the average cost difference is: $\$[6,900-300-(6,900-300) .1]-\$[2,672-500-(2,672-500) .2]=\$ 3,473.20 \%$ of this is $\$ 695$.

${ }^{49}$ The easiest way to see this is to note that in the term $e^{\gamma\left(P_{H}+O O P-\delta_{0} H-\ldots\right)}, \delta_{0} H$ and $P_{H}$ enter identically. Because men and women and younger and older consumers face the same (unobserved) price, the $\delta_{a}$ and $\delta_{F}$ coefficients are not affected when $\delta_{0}$ moves one-for-one with the assumed $P_{H}$.

${ }^{50}$ The plan $H$ intercept indicates that, overall, agents make choices as if contract $H$ were priced at $\$ 832$ more than the assumed price. There are several ways to interpret this coefficient. First and most obvious, actual employee contributions to premiums are not observed, so the assumed price difference between contracts $L$ and $H$ may be wrong, and the coefficient may partially reflect this. Alternatively, the CARA model may fail to adequately describe all the complexities of health plan choice, in which case the plan $H$ coefficient is merely a convenient modification that allows the model to better fit choice
} 
viation of idiosyncratic tastes $(\$ 1,351)$ rationalizes the observation that, within age and sex groups, many consumers with high expected spending choose the lower coverage plan and many with low expected spending choose the higher coverage plan. In terms of the model, this idiosyncratic taste parameter corresponds to the within-group heterogeneity of Figure 4. Appendix Figure A4 shows that the estimated model fits the data well, replicating the observed demand patterns of Figure 6.

The coefficient of absolute risk aversion is $6.96 \cdot 10^{-4}$. To facilitate interpretation, Table 7 compares this estimate to risk aversion estimates from other studies. Risk preferences are likely to be context-dependent, and several of the estimates listed in Table 7 are derived from other insurance markets. Nonetheless, the estimate here falls squarely within the range of the existing literature. My estimate of $\gamma$ comes closest to estimates from Handel (2013), which were also derived in a health plan context, though with a different identification strategy. To further aid interpretation, the last column in Table 7 displays a certainty equivalent measure of the risk parameters. The certainty equivalent here is the amount $X$ that would make someone just indifferent between accepting a gamble in which they win $\$ 100$ or lose $X$ with equal probability versus a status quo where nothing happens. The certainty equivalent value implied by the estimate of risk aversion here is $\$ 93$.

\section{Policy Simulations and Welfare}

In this section, I leverage the structure of Section 5 to: (i) demonstrate that the empirical within- and across-group variation in demand corresponds to the model of Figure 4, (ii) compare the optimal uniform price with the optimal age- and sex-specific prices, and (iii) generate back-of-the envelope welfare estimates under a policy counterfactual of optimal age- and sex-specific prices.

\subsection{Simulation and Connection to Theory}

I begin by simulating demand in a way that allows me to complete the link between the model and the data. Figure 7 plots the empirical analogue to the graphical model in Figure 4. To reduce clutter, I focus on comparing the 50- to 59-year-olds and the 18- to 29-year-olds, which are analogous to the $a / b$ types in Figure 4 . The insurer portion of expected healthcare costs, $c_{i j}$, are the relevant cost variables here. These are derived directly from the medical risk distributions and cost-sharing

patterns in the data. In terms of fit, a likelihood ratio test strongly rejects the null model in which the plan $H$ intercept is not included $(p<.0001)$. 
rules, as described in Sections 3.3 and 5.2. Consumer valuations are found by applying the utility function parameters from Table 6 to the out-of-pocket spending distributions implied by plan rules and medical risk. These plan valuations are measured in dollars as the certainty equivalent, $C E_{i j}$, that makes agent $i$ just indifferent between paying that sure amount and facing the uncertain loss associated with insurance plan $j .{ }^{51}$ For Panel A only, each individual in the sample is given a draw from the distribution of $\epsilon_{H} \cdot{ }^{52}$

Panel A of Figure 7 plots plan valuations versus costs. Each scatterpoint represents an observation. Because the choice in this setting is between two plans (greater or lesser insurance), the relevant valuations and costs are the differences in valuations and costs betweens plans: $C E_{i H}-C E_{i L}$ and $c_{i H}-c_{i L}$. The 45-degree line is plotted for reference. Only for the cases in which $C E_{i H}-C E_{i L}$ exceeds $c_{i H}-c_{i L}$ is the choice of plan $H$ socially efficient. ${ }^{53}$

Panel B of Figure 7 plots the demand curves and cost "clouds" that correspond to Panel A. To simulate demand, I calculate the person-specific probability of enrollment in plan $H$ at each price level, denoted $D_{i}\left(P_{H}\right)$. As above, $P_{H}$ is the premium difference between $L$ and $H$. Demand for $H$ is calculated as the indicator function for taking it up, integrated over the taste distribution:

$$
D_{i}\left(P_{H}\right)=\int \mathbf{1}\left(V_{i H}>V_{i L} \mid \epsilon, P_{H}\right) \cdot d F\left(\epsilon_{H}\right)
$$

This takeup is summed over individuals to generate the demand curve. Insurer expected cost clouds are plotted along with demand. To keep the plot readable, I sample 50 cost points $\left(c_{i H}-c_{i L}\right)$ for each price level on the demand curve, using sampling probabilities equal to $D_{i}\left(P_{H}\right)$. This generates a cloud corresponding to the average cost curve. I also "jitter" the points because many of the expected cost differences overlap. The level of the cost curve is low, and its slope is shallow because the insurer's spending difference between plans is only about $10 \%$ of spending-the difference in coinsurance

\footnotetext{
${ }^{51}$ Specifically, $C E_{i j}$ is found by equating the utility of the sure amount to the expected utility of the lottery, and solving for the sure amount: $-\frac{1}{\gamma} e^{-\gamma \cdot C E_{i j}}=\int-\frac{1}{\gamma} e^{\gamma \cdot\left(P_{j}+O O P-\delta_{0} H-\sum_{a} \delta_{a} I_{a} \times H-\delta_{F} I_{F} \times H+\epsilon_{i H}\right)} d F_{i j}(O O P)$.

${ }^{52}$ In Panel B, I integrate over the error distribution to generate expected demand for each individual at each price.

${ }^{53}$ To put the dollar amounts represented along the horizontal axis in context, recall that the relevant marginal cost from a social welfare perspective is the difference in the insurer's expected cost of enrolling the consumer in plan $H$ instead of plan $L$. For a consumer with the average level of healthcare charges, $\$ 3,865$, this marginal cost would be approximately $\$ 220$. $\operatorname{MC}(\$ 3,865)=c_{H}(\$ 3,865)-c_{L}(\$ 3,865)=\$[3,865-300-0.9 \times(3,865-300)]-\$[3,865-500-0.8 \times(3,865-500)]=\$ 220$. The simple calculation in this note ignores the concavity of cost-sharing rules, applying the cost-sharing rules to the expected level of healthcare utilization rather than taking the expectation over the distribution of insurer costs. The actual calculation of the marginal cost curves in Figure 7 fully accounts for the non-linearity of cost-sharing rules and integrates plan costs over the individual-specific health risk distribution.
} 
rates.

Figure 7 closely parallels the model of Figure 4. In Panel A of Figure 7, valuation-cost pairs are dispersed within and across groups. The scatterpoints span the 45-degree line differentially for the two age groups, exactly as in Panel A of Figure 4. The residual within-group heterogeneity apparent in the figure highlights that price discrimination on age represents a feasible welfare improvement, but not a first-best, as there is significant heterogeneity that is not (and possibly cannot be) priced. Panel B of Figure 7 likewise mirrors Panel B of Figure 4, showing within- and across-group demand heterogeneity, reflected in distinct demand curves for the two age groups and in the cost clouds. This feature that costs do not trace a single line is special to selection markets, where the costs are a function not of some producers' production technology, but are rather a function of the buyers' cost types.

With respect to optimal prices, the demand-cost intercepts for the two age groups will clearly differ. Older consumers in this market optimally face higher prices and demand more insurance at those higher prices, while younger consumers optimally face lower prices and demand less insurance at those lower prices. This matches the model in Figure 4, but stands in stark contrast to conventional wisdom, which would imply that young and old would differ in demand only because they differed in insurable risk, and which would predict that takeup conditional on insurable risk would be identical across the two groups. ${ }^{54}$

\subsection{Optimal Pricing Counterfactuals}

I next turn to optimal pricing and welfare. The welfare estimates are, of course, specific to the empirical setting, so at the end of this section I discuss briefly how the welfare costs of non-discrimination regulations might differ in other insurance market contexts.

Social surplus is defined following Equation (4) and is the certainty equivalent valuation of the chosen plan minus the insurer's cost of providing coverage in that plan. Optimal prices are those that maximize social surplus by inducing consumers to choose the plan that generates the greatest utility net of costs. ${ }^{55}$ The constrained optimal uniform price is found at the intersection of demand and

\footnotetext{
${ }^{54}$ For comparison, Appendix Figure A1 draws the type of demand homogeneity across consumer types that is almost always implicitly assumed in the literature. In that figure, a single price sorts efficiently since consumer types (men and women, young and old, rich and poor) differ in demand on average only because they differ in insurable risk on average.

${ }^{55}$ In markets with heterogeneity, marginal costs are better described as clouds than curves, so rather than attempting to graphically identify a single intersection point within that cloud, optimality is determined by numerically searching over prices and identifying the price that maximizes social surplus.
} 
marginal cost in the market overall, while the constrained optimal group-specific prices are found at the corresponding group-specific intersection. I consider these optimal pricing policies, rather than equilibrium pricing, to focus on the novel result of the paper: that optimal pricing as a function of observables generates a feasible welfare improvement over optimal uniform pricing. Nonetheless, all of the prices considered could be implemented as a competitive market equilibrium by a regulator setting appropriate consumer subsidies or tax credits. ${ }^{56}$

Panel A of Table 8 describes a baseline case in premiums equal to $20 \%$ of the average insurer cost difference between plans in the observed equilibrium (\$695). This level of subsidization is typical for a large employer, but it could also result from imperfect risk adjustment in a competitive market setting. In the remaining panels, prices, takeup rates, and welfare gains under counterfactuals are compared to this baseline. Panel B implements constrained optimal uniform pricing and Panels $\mathrm{C}$ and D implement constrained optimal pricing by age and sex, respectively. Throughout all the counterfactuals, only prices-not the menu of options-are assumed to change, so that only takeup and costs respond in equilibrium.

In Panels B through D, columns (1) and (2) report the counterfactual price and the quantity at that price, columns (3) and (4) report the changes in price and quantity relative to baseline, and column (5) reports the corresponding change in social surplus. This social surplus measure incorporates both buyer's and seller's welfare. These differences from baseline are presented overall, as well as within age and sex groups to gauge incidence.

Consistent with the model in Section 2, the reduced form evidence in Section 4, and as suggested by the simulation displayed in Figure 7, Table 8 shows that there is a feasible welfare improvement in allowing price discrimination by age or sex, even relative to constrained optimal uniform prices. These can be seen comparing prices in Panels B versus C, or B versus D. The youngest group of consumers optimally faces prices that are \$205 less than those optimally faced by the oldest group in the data. Additionally, women optimally face a price that is $\$ 100$ larger than the price that optimally sorts men. This implies that the price that sorts men efficiently will lead to women being overinsured, and the price that sorts women efficiently will lead to men being underinsured. If-counter to fact,

\footnotetext{
${ }^{56}$ There is no important conceptual difference between an employer or social planner who sets the efficient incremental cost for enrolling in $H$ and the regulator who implements the same efficient price differential in a competitive market setting via risk adjustment and/or subsidizing premiums. In the constrained optimal uniform pricing scenario, the directto-consumer subsidies are assumed to be uniform. Otherwise, the subsidies would be effectively generating different prices across the consumer groups, in violation of the uniform price constraint.
} 
but consistent with the standard assumption implicit in the literature-these groups had identical demand conditional on objective risk, then no improvement would be feasible relative to the optimal uniform price. The fact that optimal prices differ is because demand (conditional on costs) differs across the demographic groups.

Moving from Panel A to B, the resulting gains from optimal uniform pricing, \$24/person on average over the population, are comparable in size to other estimates in the literature of the welfare loss of observed pricing relative to optimal uniform pricing in employer plans (Carlin and Town, 2008; Einav, Finkelstein and Cullen, 2010; Bundorf, Levin and Mahoney, 2012). Moving from Panel B to $C$ or from $B$ to $D$, the incremental welfare gains of adopting optimal group-specific prices are an order of magnitude smaller.

The welfare gains associated with implementing group-specific prices relative to optimal uniform prices may be small here because the difference in financial insurance between the two plan options considered here is minor. This leads to optimal uniform and optimal group-specific prices that are on the order of a few hundred dollars apart. To gain some intuition for the result, note that a reasonable approximation of the welfare loss of suboptimal pricing is $-\frac{1}{2} \cdot\left(Q^{*}-Q\right) \cdot\left(P^{*}-P\right)$, where $\left(Q^{*}, P^{*}\right)$ are the optimal price and quantity. Comparing Panels B and $\mathrm{C}$, enrollment for the 18- to 29 -year-olds increases by 2.7 percentage points $(11 \%)$, and prices decline by $\$ 110$. Therefore, it is unsurprising to observe average welfare gains of optimally price-discrimating on the order of $\$ 1.50\left(\approx \frac{1}{2} \cdot \$ 110 \cdot 0.027\right)$. Intuitively, the potential welfare losses in this market are limited by the the assumption that the consumer must choose either plan $L$ or plan $H$, either of which provide generous financial insurance.

By the same logic, the welfare losses could be considerably higher in a setting in which remaining uninsured were a relevant outside option. To see this, consider that the average of the marginal cost difference between the plans $c_{i H}-c_{i L}$ for the youngest and oldest groups are, respectively, \$134 $\left(=E\left[c_{i H}-c_{i L} \mid\right.\right.$ age $\left.\left.18-29\right]\right)$ and $\$ 360\left(=E\left[c_{i H}-c_{i L}\right.\right.$ lage 50-59]). These averages are not far from the optimal prices, which must lie somewhere along the marginal cost clouds. Now consider a hypothetical choice between plan $H$ and no insurance. Here the marginal cost difference would be $\$ 743$ $\left(=E\left[c_{i H}-0 \mid\right.\right.$ age $\left.\left.18-29\right]\right)$ for the younger group and $\$ 3,850\left(=E\left[c_{i H}-0 \mid\right.\right.$ age $\left.\left.50-59\right]\right)$ for the older group. If these averages were likewise not far from the optimal prices, then it is plausible that these prices could differ by around $\$ 3,000$. This level of difference, which is related to the height of the 
welfare loss triangle, could imply significantly greater welfare loss from uniform pricing. ${ }^{57}$ In any case, it is important to at least note that the magnitude of the welfare losses in Table 8 are completely context-specific. The general lesson is that demand heterogeneity across identifiable groups implies a feasible improvement by pricing separately.

\section{Implications and Conclusion}

Today, US employers are barred by ERISA and HIPAA from setting premium contributions for employee health plans that differ on the basis of age, sex, or almost any other observable worker characteristic. In the health insurance Marketplaces established by the Affordable Care Act, price discrimination on age is allowed with binding restrictions, and price discrimination on sex is outlawed. In the US Medicare system, insurers offering private managed care plans under the Medicare Advantage program must offer plans to all eligible beneficiaries at identical prices within a local market, without regard to consumer characteristics like age, sex, and-except for Medicaid eligibility—wealth or income. And throughout all of the European Union, in health insurance, auto insurance, annuities, and life insurance, insurers are barred from setting different premiums for men and women. The regulatory trend is clearly towards continued and expanded equity in pricing, with policymakers in the US and abroad continuing to voice support for such policies. ${ }^{58}$

The conventional wisdom holds that with the proper tools like risk adjustment and consumer subsidies, regulators can undo the selection problem created by these non-discrimination policies and generate the best feasible allocation. The new insight of this paper is that this notion is incorrectprice discrimination on observables yields a feasible improvement. This equity-efficiency tradeoff is a fundamental feature of insurance markets with demand heterogeneity, and there is little reason to assume that demand heterogeneity is not an important part of most insurance market settings. While regulators and policymakers may nonetheless wish to pursue equity objectives, there is an unavoidable efficiency cost of doing so.

\footnotetext{
${ }^{57}$ The demand estimation above is not appropriate for the task of simulating this loss directly. While the risk aversion parameter is a primitive of the utility function that could be applied to the choice of plan $H$ versus no insurance, the $\delta$ and $\epsilon$ parameters are preferences that are specific to the choice between plan $L$ and plan $H$.

${ }^{58}$ President Obama has repeatedly framed the issue as one of fairness. For example: "We passed the Affordable Care Act, which ... says women can't be charged more for health insurance just because they're women" (Barack Obama, March 16, 2016). The EU's Justice Commissioner has made similar remarks: “Gender equality is a fundamental right in the European Union and the Court of Justice made clear that this also applies to insurance pricing" (European Commission, December 20, 2012).
} 
With the caveat that the parameters and welfare estimates here are specific to the setting I study, I find that optimal age-specific prices increase in age, and they are higher for women than for men. With respect to age-based pricing, it is interesting to note that in the heavily regulated ACA Marketplaces, prices follow a schedule that is increasing in age, matching in a superficial sense the optimal age pattern I estimate here. The age-varying price schedule implemented in the Marketplaces was most likely motivated by a desire to reduce the implicit transfers from younger to older consumers rather than by efficiency concerns. The prima facie similarity to an optimally set schedule is coincidental.

There is an important role for future work to uncover what drives the striking demand differences by age and sex uncovered here. In the first steps toward this exploration taken in this paper, demand heterogeneity appears not be driven by differences in higher moments of the risk distribution, by differential inertia across young and old or men and women, by subject beliefs based on last-period heuristics, or by differential private information about future healthcare costs.

Another important task for future work will be to apply the ideas here to other observable consumer types-e.g., income-based pricing, as in Glazer and McGuire (2011) or prices linked to the dispersion of risk consumers face. Perhaps most importantly, future work should consider how acknowledging demand heterogeneity affects optimal regulatory policy in other markets, and in particular, markets in which remaining uninsured is a relevant potential outcome. In such settings, welfare losses arising from uniform pricing could be significant. 


\section{References}

Abaluck, Jason, and Jonathan Gruber. 2011. "Choice Inconsistencies among the Elderly: Evidence from Plan Choice in the Medicare Part D Program." American Economic Review, 101: 1180-1210.

Akerlof, George A. 1970. "The Market for "Lemons": Quality Uncertainty and the Market Mechanism." The Quarterly Journal of Economics, 84(3): pp. 488-500.

Angrist, Joshua D, and Jörn-Steffen Pischke. 2008. Mostly harmless econometrics: An empiricist's companion. Princeton university press.

Breyer, Friedrich, M Kate Bundorf, Mark V Pauly, et al. 2011. "Health care spending risk, health insurance, and payment to health plans." Handbook of Health Economics, 2: 691-762.

Bundorf, M. Kate, Jonathan Levin, and Neale Mahoney. 2012. "Pricing and Welfare in Health Plan Choice." American Economic Review, 102(7): 3214-48.

Carlin, Caroline, and Robert Town. 2008. "Adverse Selection, Welfare and Optimal Pricing of Employer-Sponsored Health Plans." mimeo, University of Minnesota.

Chetty, Raj, and Amy Finkelstein. 2013. "Chapter 3 - Social Insurance: Connecting Theory to Data." In handbook of public economics, vol. 5. Vol. 5 of Handbook of Public Economics, , ed. Martin Feldstein Alan J. Auerbach, Raj Chetty and Emmanuel Saez, 111 - 193. Elsevier.

Chiappori, Pierre-André, and Bernard Salanie. 2000. "Testing for asymmetric information in insurance markets." Journal of political Economy, 108(1): 56-78.

Cohen, Alma, and Liran Einav. 2007. "Estimating Risk Preferences from Deductible Choice." The American Economic Review, 97(3): pp. 745-788.

Cutler, David M., Amy Finkelstein, and Kathleen McGarry. 2008. "Preference Heterogeneity and Insurance Markets: Explaining a Puzzle of Insurance." The American Economic Review, 98(2): pp. 157-162.

Cutler, David M., and Sarah J. Reber. 1998. "Paying for Health Insurance: The Trade-Off between Competition and Adverse Selection." The Quarterly Journal of Economics, 113(2): 433-466.

Einav, Liran, Amy Finkelstein, and Jonathan Levin. 2010. "Beyond Testing: Empirical Models of Insurance Markets." Annual Review of Economics, 2(1): 311-336.

Einav, Liran, Amy Finkelstein, and Mark R. Cullen. 2010. "Estimating Welfare in Insurance Markets Using Variation in Prices." The Quarterly Journal of Economics, 125(3): 877-921.

Einav, Liran, Amy Finkelstein, Stephen P Ryan, Paul Schrimpf, and Mark R Cullen. 2013. "Selection on moral hazard in health insurance." The American economic review, 103(1): 178-219.

Einav, Liran, and Amy Finkelstein. 2011. "Selection in Insurance Markets: Theory and Empirics in Pictures." Journal of Economic Perspectives, 25(1): 115-38.

Ericson, Keith M Marzilli, and Amanda Starc. 2015. "Pricing regulation and imperfect competition on the massachusetts health insurance exchange." Review of Economics and Statistics, 97(3): 667-682.

Fang, Hanming, Michael P. Keane, and Dan Silverman. 2008. "Sources of Advantageous Selection: Evidence from the Medigap Insurance Market." Journal of Political Economy, 116(2): pp. 303-350. 
Feldman, Roger, and Bryan Dowd. 2000. "Risk Segmentation: Goal or Problem?" Journal of Health Economics, 19(4): 499 - 512.

Finkelstein, Amy, and James Poterba. 2004. "Adverse selection in insurance markets: Policyholder evidence from the UK annuity market." Journal of Political Economy, 112(1): 183-208.

Finkelstein, Amy, and Kathleen McGarry. 2006. "Multiple Dimensions of Private Information: Evidence from the Long-Term Care Insurance Market." The American Economic Review, 96(4): pp. 938958.

Finkelstein, Amy, James Poterba, and Casey Rothschild. 2009. "Redistribution by insurance market regulation: Analyzing a ban on gender-based retirement annuities." Journal of Financial Economics, 91(1): $38-58$.

Glazer, Jacob, and Thomas G. McGuire. 2000. “Optimal Risk Adjustment in Markets with Adverse Selection: An Application to Managed Care." American Economic Review, 90(4): 1055-1071.

Glazer, Jacob, and Thomas G McGuire. 2011. "Gold and Silver health plans: Accommodating demand heterogeneity in managed competition." Journal of health economics, 30(5): 1011-1019.

Hackmann, Martin B, Jonathan T Kolstad, and Amanda Ellen Kowalski. 2015. "Adverse Selection and an Individual Mandate: When Theory Meets Practice." The American Economic Review, 105(3): 1030-1066.

Handel, Ben, Igal Hendel, and Michael D. Whinston. 2015. "Equilibria in Health Exchanges: Adverse Selection versus Reclassification Risk." Econometrica, 83(4): 1261-1313.

Handel, Benjamin R. 2013. "Adverse selection and inertia in health insurance markets: When nudging hurts." The American Economic Review, 103(7): 2643-2682.

Handel, Benjamin R., Jonathan T. Kolstad, and Johannes Spinnewijn. 2015. "Information Frictions and Adverse Selection: Policy Interventions in Health Insurance Markets." National Bureau of Economic Research Working Paper 21759.

Kaiser Family Foundation and the Health Research and Educational Trust. 2007. "Annual Survey of Employer Health Benefits, 2007."

Layton, Timothy. 2014. "Imperfect Risk Adjustment, Risk Preferences, and Sorting in Competitive Health Insurance Markets." Working paper.

Layton, Timothy J., Randall P. Ellis, and Thomas G. McGuire. 2015. “Assessing Incentives for Adverse Selection in Health Plan Payment Systems." National Bureau of Economic Research Working Paper 21531.

Mahoney, Neale. 2015. "Bankruptcy as Implicit Health Insurance." The American Economic Review, 105(2): 710-746.

Manning, Willard G, Joseph P Newhouse, Naihua Duan, Emmett B Keeler, and Arleen Leibowitz. 1987. "Health insurance and the demand for medical care: evidence from a randomized experiment." The American economic review, 251-277.

Rothschild, Michael, and Joseph Stiglitz. 1976. "Equilibrium in Competitive Insurance Markets: An Essay on the Economics of Imperfect Information." The Quarterly Journal of Economics, 90(4): pp. 629-649. 
Starc, Amanda. 2014. "Insurer pricing and consumer welfare: Evidence from medigap." The RAND Journal of Economics, 45(1): 198-220.

Train, Kenneth. 2009. Discrete Choice Methods With Simulation. Cambridge University Press.

Van de Ven, Wynand P.M.M., and Randall P. Ellis. 2000. "Risk adjustment in competitive health plan markets." In Handbook of Health Economics. Vol. 1 of Handbook of Health Economics, , ed. A. J. Culyer and J. P. Newhouse, Chapter 14, 755-845. Elsevier. 


\section{Figure 1: Selection -or- Demand Heterogeneity: A Single Price Can Sort Efficiently}

(A) demand heterogeneity without selection $(v, c)$

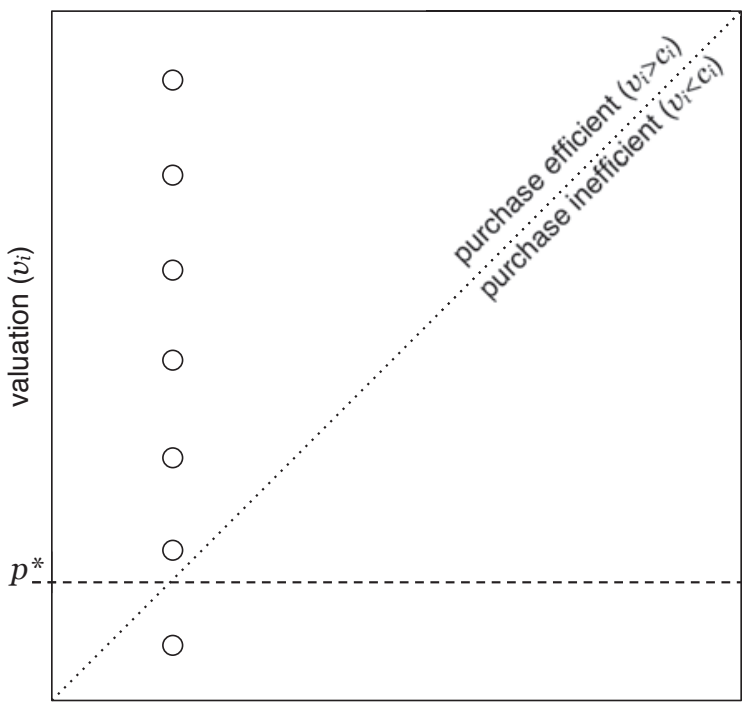

marginal cost $\left(c_{i}\right)$

(C) demand heterogeneity without selection $(\mathrm{P}, \mathrm{Q})$

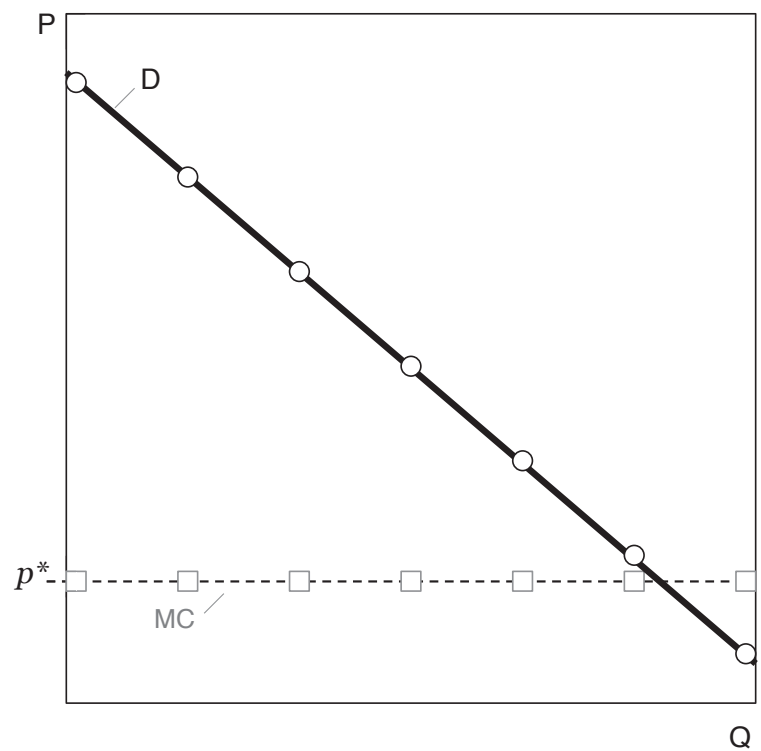

(B) selection without heterogeneity $(v, c)$

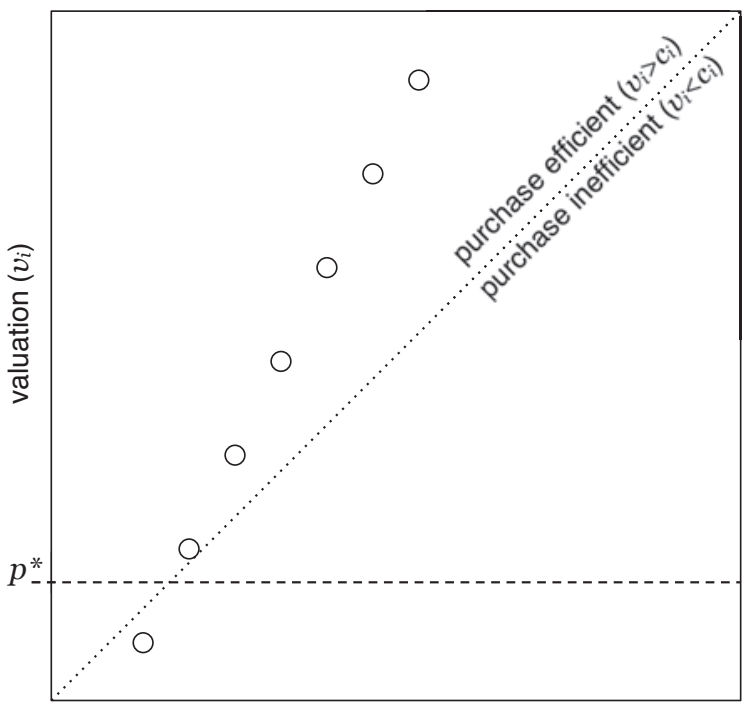

marginal cost $\left(c_{i}\right)$

(D) selection without heterogeneity $(\mathrm{P}, \mathrm{Q})$

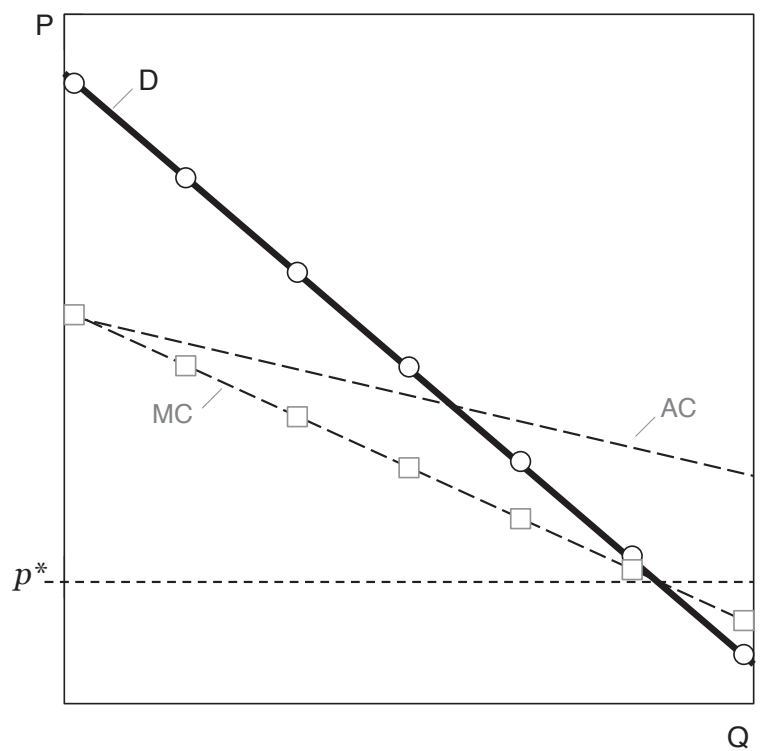

Note: Figure establishes the baseline cases of demand heterogeneity without selection (Panels A and C) and of selection without demand heterogeneity (Panels B and D). Circles represent individuals, with the vertical axes in the top panels measuring plan valuation, $v$, and the horizontal axes measuring the insurer's expected costs of covering claims, c. Selection implies correlation between $c$ and $v$. Panels $C$ and D plot the demand and cost curves implied by the cost and valuation pairs plotted in Panels A and B. Valuations are plotted as circles and costs as squares in the bottom panels, and quantity along the horizontal axes is scaled from 0 to $100 \%$. Demand heterogeneity is defined as consumer valuations that vary after conditioning on the insurer's expected costs. In these plots, this would imply multiple vertical positions for some fixed horizontal position in $(v, c)$ space, as in Panel A. The $45-$ degree line separates the cases in which purchasing insurance is socially efficient from those in which it is efficient to remain uninsured. Consumers make efficient choices if and only if $v \geq c$, and choose to take up insurance if and only if $v \geq p$. In these baseline cases, a single price $p^{*}$ sorts all consumers efficiently. 


\section{Figure 2: Selection -and- Demand Heterogeneity: No Single Price Sorts Efficiently}

(A) selection and heterogeneity $(v, c)$

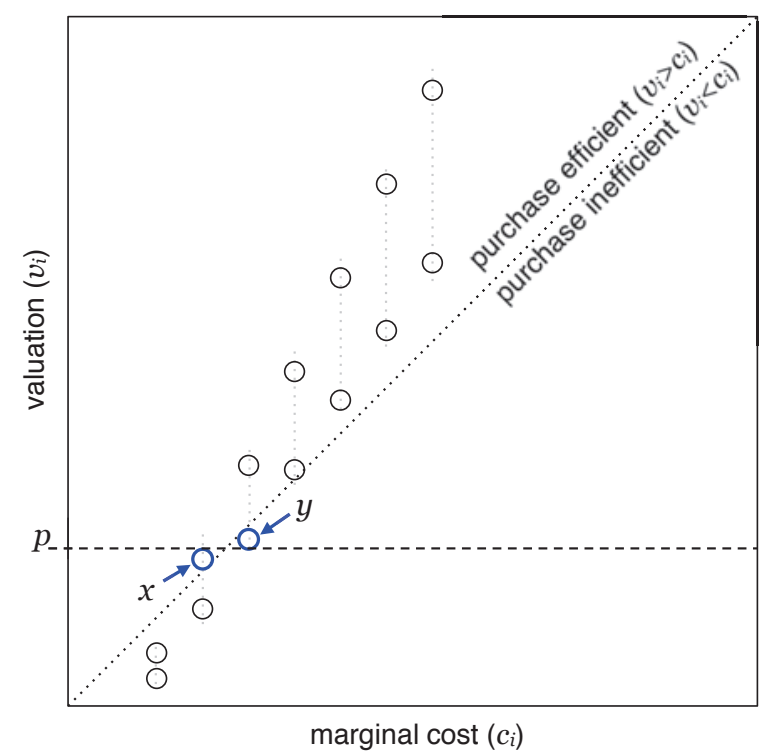

(B) selection and heterogeneity $(\mathrm{P}, \mathrm{Q})$

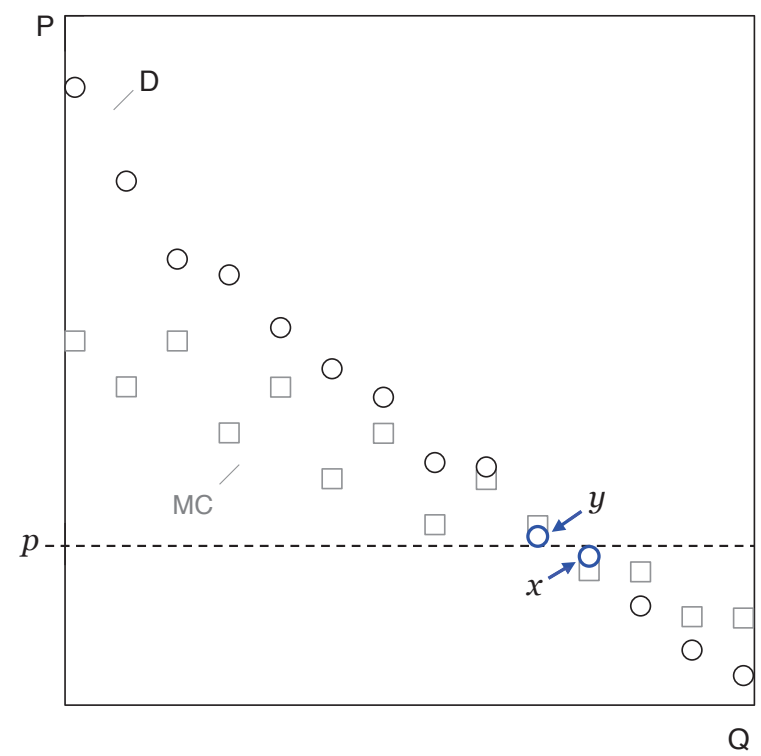

Note: Figure shows how selection and demand heterogeneity interact in selection markets. The panels are constructed as in Figure 1, but now simultaneously incorporate demand heterogeneity and selection. Under these conditions, no single price can sort all consumers efficiently. Price would need to be higher than the depicted price $p$ to sort consumer $y$ efficiently, but lower than $p$ to sort consumer $x$ efficiently. In the corresponding demand diagram of Panel B, demand declines monotonically, but the costs implied by Panel A lead to a non-monotonic marginal cost "cloud" because there is no longer a one-to-one mapping of consumer valuations to marginal costs. See the notes to Figure 1 for additional documentation. 
Figure 3: Price Discrimination on Preferences Can Be Welfare-Improving, Even if Costs are Identical

(A) groups with identical costs, differing demand $(v, c)$

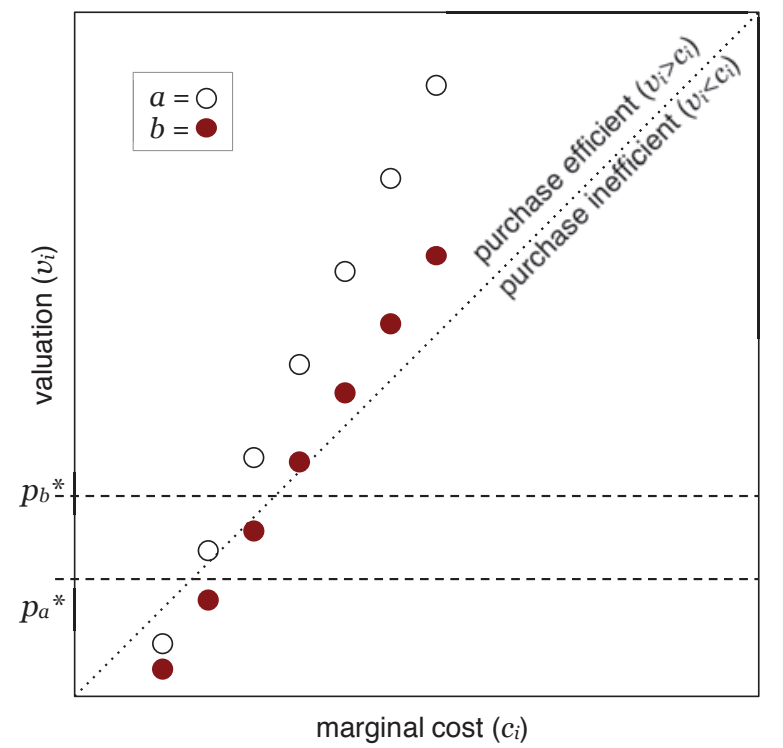

(B) corresponding demand diagrams $(\mathrm{P}, \mathrm{Q})$

(C) analogous case for continuous distribution
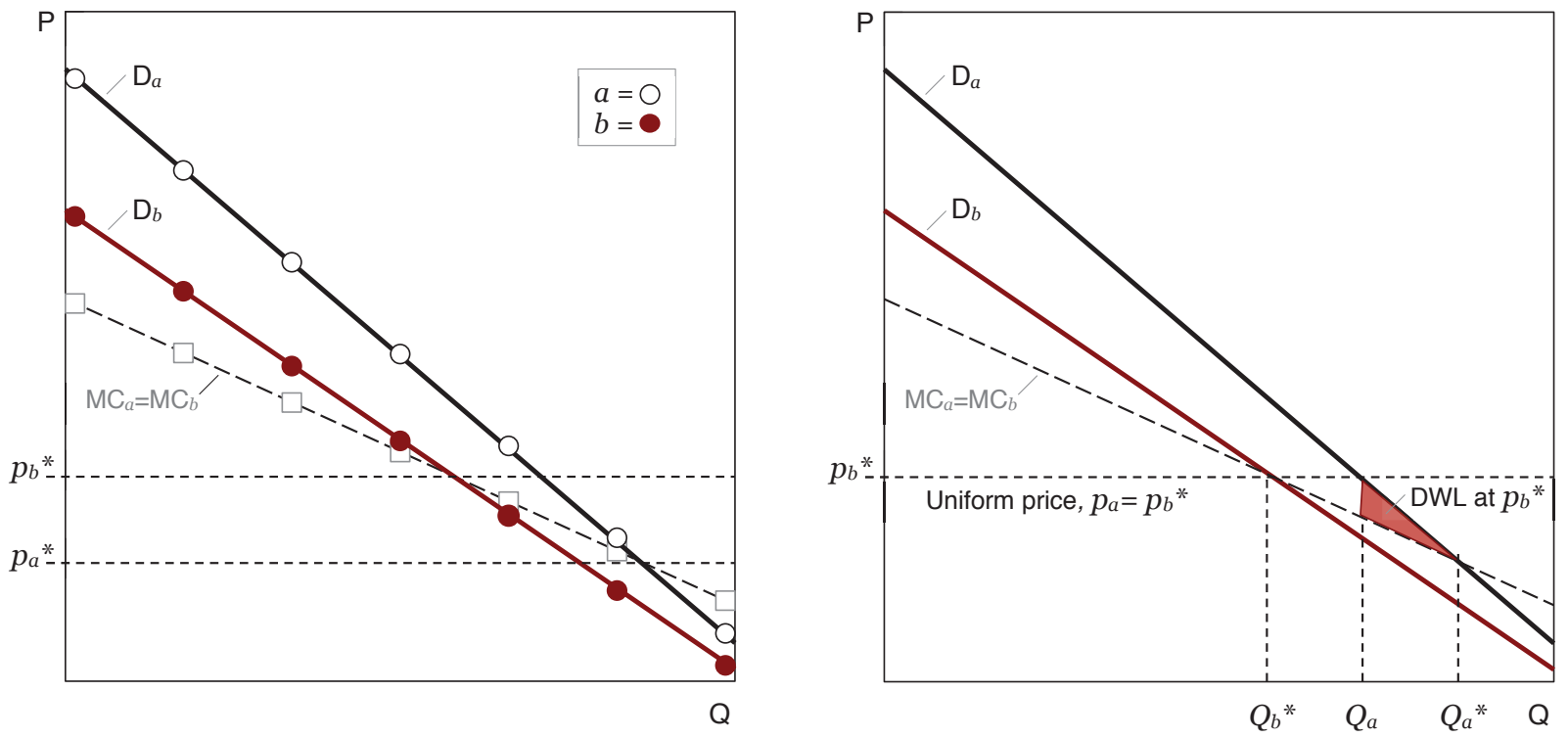

Note: Figure modifies Figure 2 to allow for identifiable consumer types, labeled $a$ and $b$, while maintaining Figure 2 's exact distribution of $(v, c)$ pairs overall. In the empirical application, identifiable types are defined by sex and age, which vary in both willingness-to-pay and costs. For simplicity, the graphical example here is constructed so that the types vary only in willingness-to-pay, $v$, and generate identical distributions of costs. Prices $p_{a}^{*}$ and $p_{b}^{*}$, respectively, sort types $a$ and $b$ efficiently. Thus price discrimination represents a feasible improvement over the best uniform price from Figure 2. Panel C plots the demand diagram for case like Panel B, but with a large set of consumers and a continuous distribution of costs within each type. In Panel C, the shaded triangle depicts the welfare loss associated with setting a uniform price for all consumers at the level that is optimal for the $b$ types, $p_{b}^{*}$. See the notes to Figures 1 and 2 for additional documentation. 


\section{Figure 4: Extension: Demand Heterogeneity Within and Across Groups}

(A) heterogeneity within and across $a / b$ groups $(v, c)$

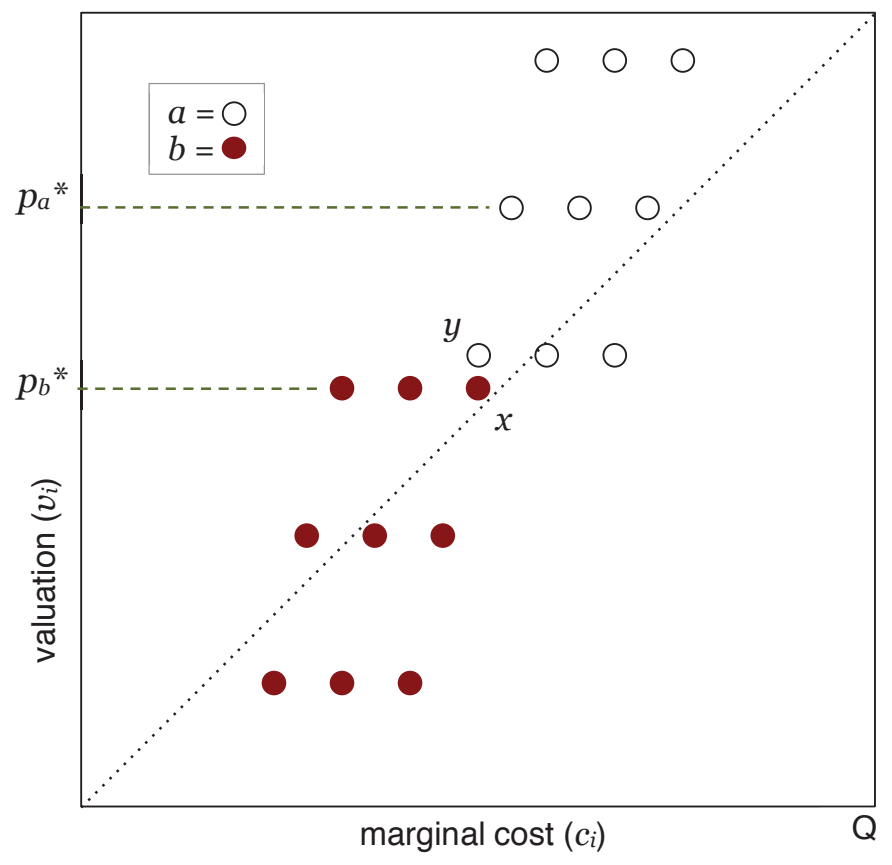

(B) corresponding demand and MC clouds $(\mathrm{P}, \mathrm{Q})$

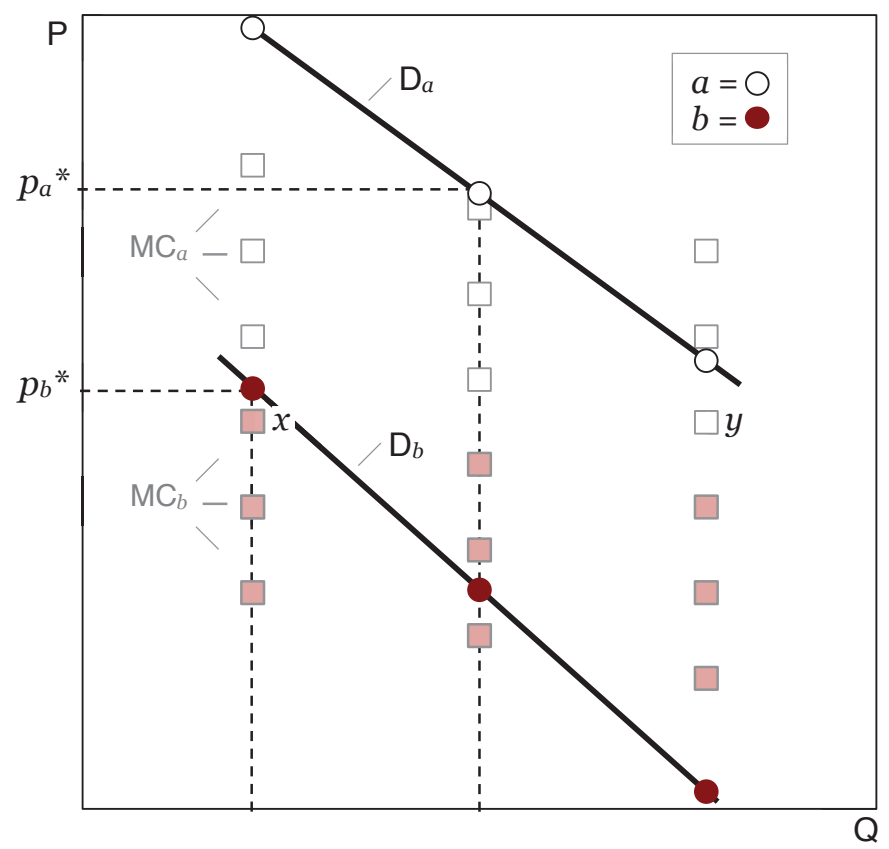

Note: Figure extends the intuition of Figure 3 to show how optimal prices are affected by allowing for both withinand across- type demand heterogeneity. The within-type demand heterogeneity introduced in the figure implies that within each of the $a$ and $b$ types, consumers facing the same expected costs have differing willingness-to-pay. Across-type demand heterogeneity can be seen by comparing the $a$ (hollow) and $b$ (solid) points, similar to Figure 3. In the corresponding demand diagram of Panel B, willingness-to-pay is shown with circles, costs with squares. As in the case where there is only across-type heterogeneity in Figure 3, no single price generates the best feasible allocation. Here, separate pricing along the $a / b$ type leads to more individuals sorted efficiently relative to uniform pricing, even though there remains residual unpriced heterogeneity that rules out a first-best solution. See the notes to Figures 1 through 3 for additional documentation. 


\section{Figure 5: Summary Statistics: Distributions of Realized Healthcare Costs}

(A) healthcare spending by age
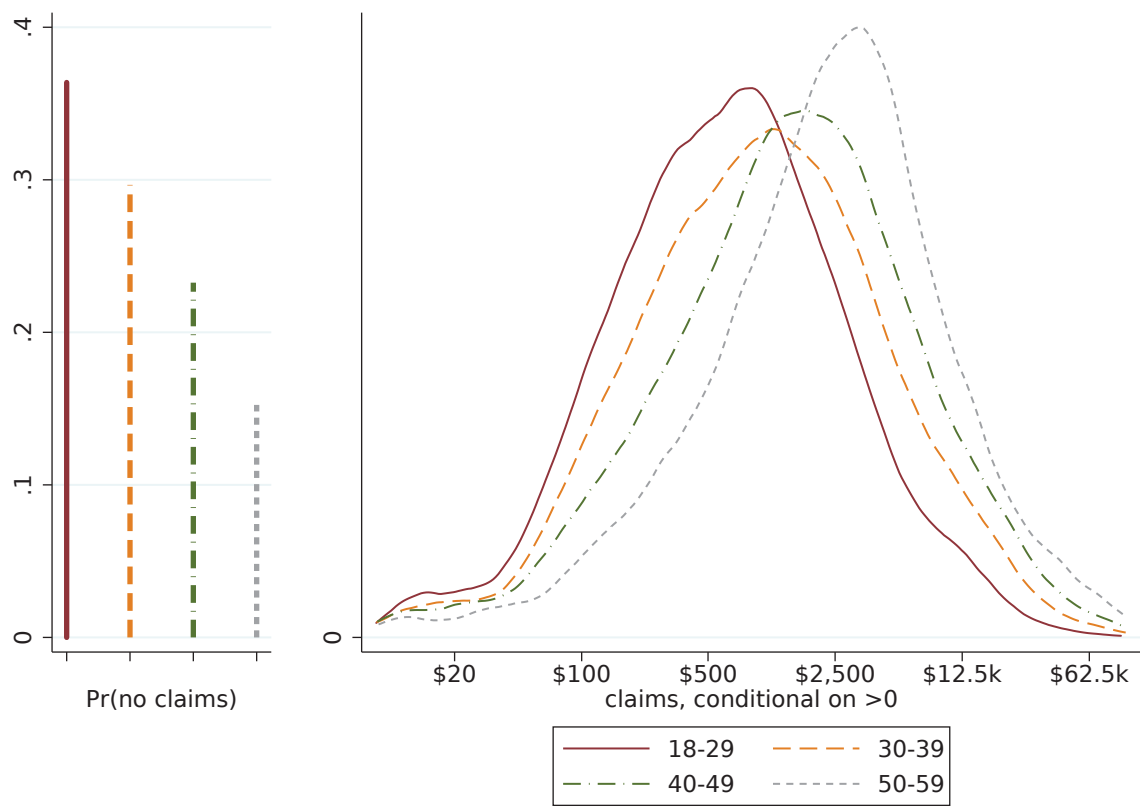

(B) healthcare spending by sex
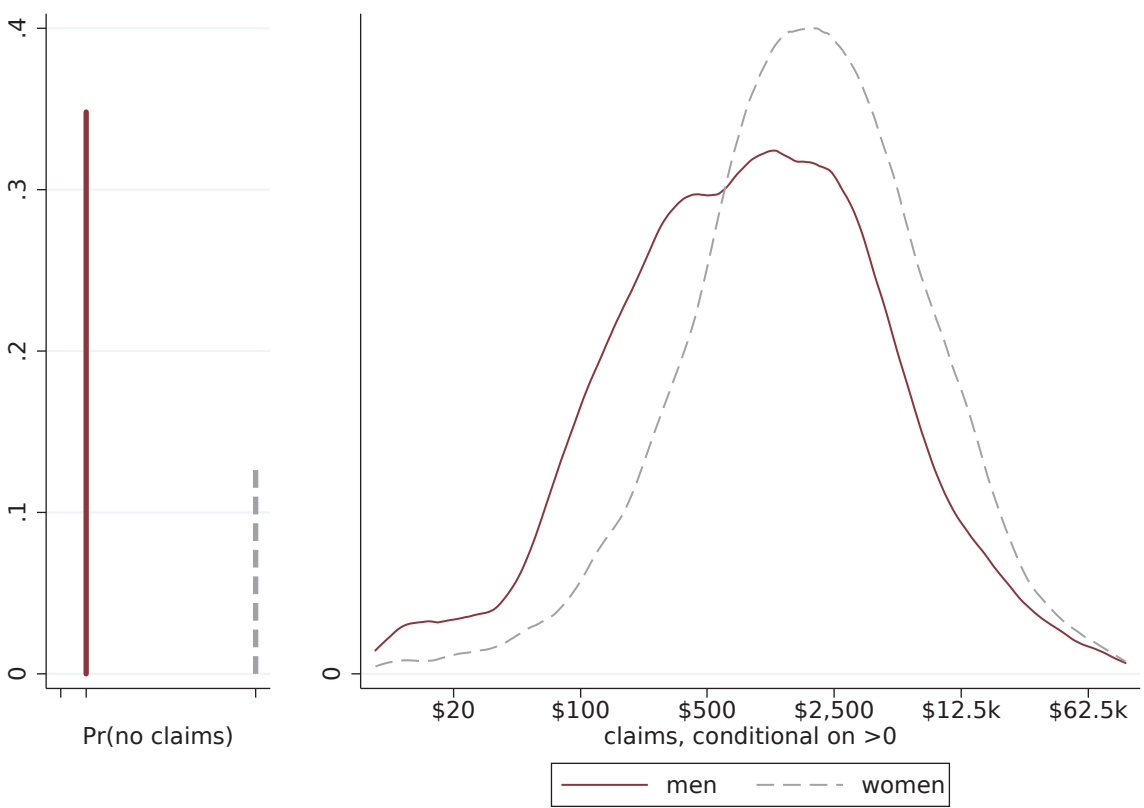

Note: Figure shows the distributions of realized healthcare spending by age (Panel A) and by sex (Panel B). The left side of each panel indicates the fraction of enrollees with no claims in the plan year-i.e., no contacts with a healthcare provider. The right side of each panel plots a kernel density estimate of the total healthcare spending in the plan year (insurer plus consumer shares), conditional on positive spending. 


\section{Figure 6: Empirical Analogue of Figure 3: Demand Heterogeneity Across Groups}

(A) groups defined by age; total spending

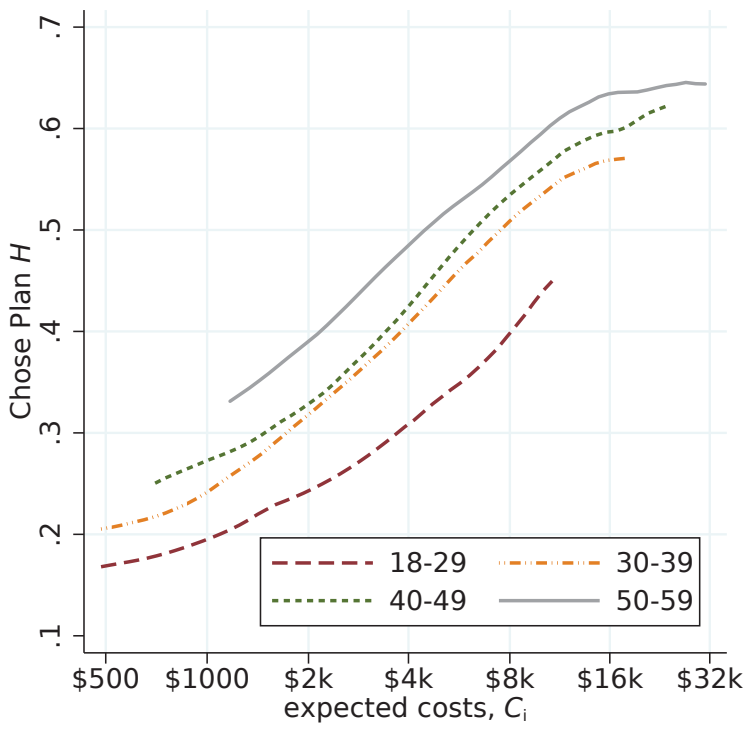

(C) groups defined by sex; total spending

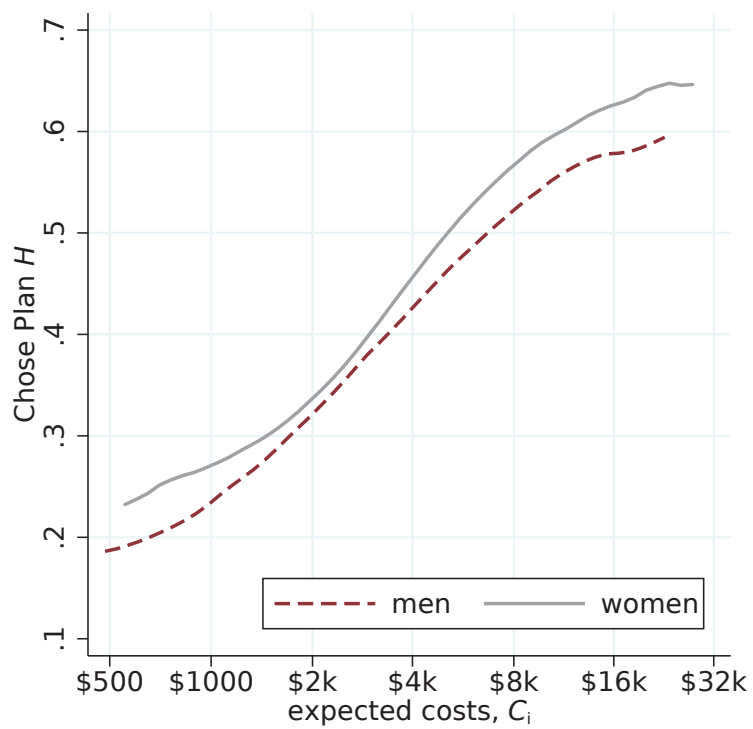

(B) groups defined by age; insurer MC

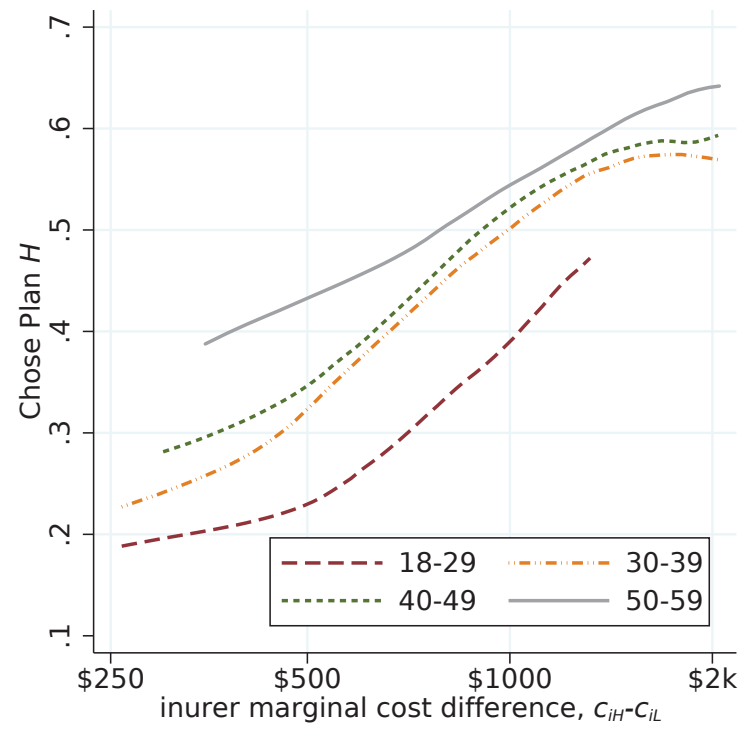

(D) groups defined by sex; insurer MC

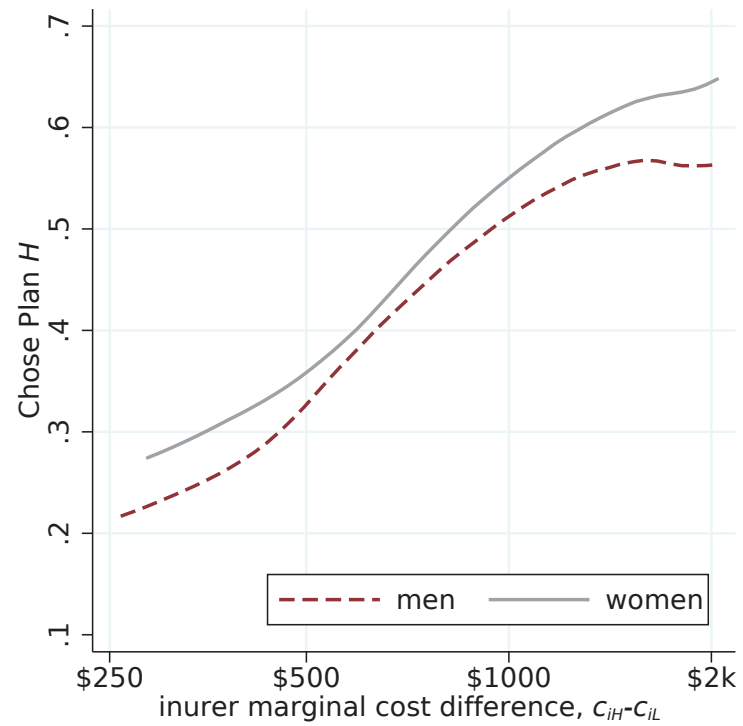

Note: Figure plots local polynomial regressions of plan choice on measures of expected healthcare consumption. In the top panels, the regressions are estimated separately in four age bins. In the bottom panels, the regressions are estimated separately by sex. The conditioning cost variable used in Panels A and C (left) is the total expected healthcare consumption, $\widehat{C}_{i}$. The cost variable used in Panels B and D (right) is the insurer's expected cost of providing plan $H$ minus the insurer's expected cost of providing plan $L$ to the same individual $\left(\widehat{c}_{i H}-\widehat{c}_{i L}\right)$. Horizontal axes are scaled in logs. The regressions underlying these plots correspond to the sufficient statistics test described in Equations (5) and (6). 
Figure 7: Empirical Analogue of Figure 4: Within- and Across-Group Demand Heterogeneity

(A) heterogeneity within and across age groups

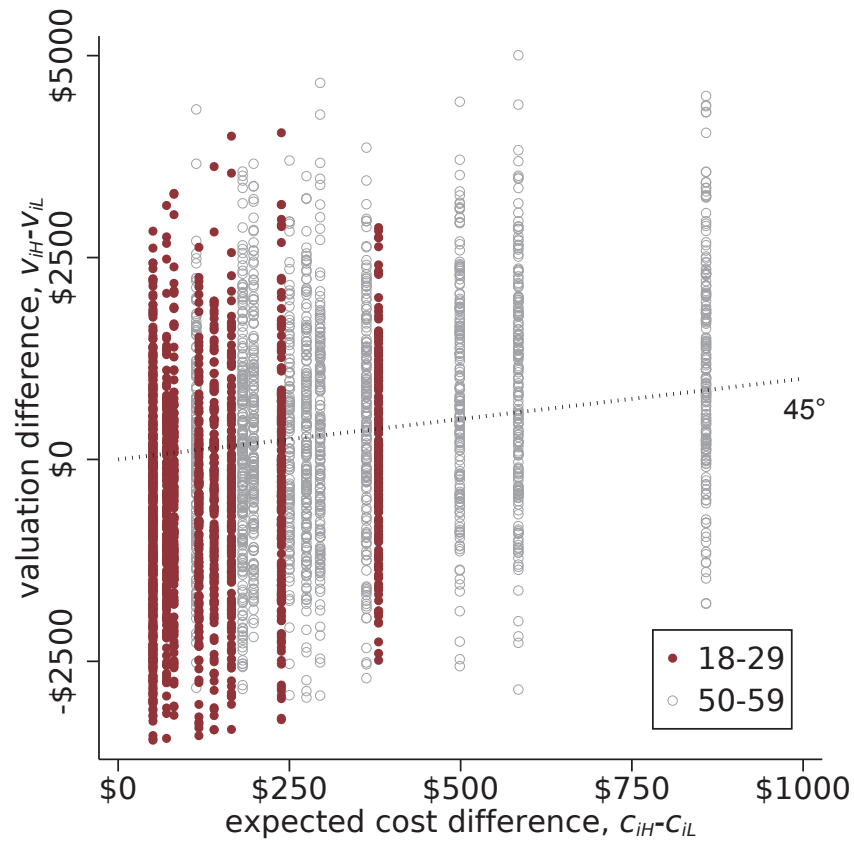

(B) corresponding demand and cost "clouds"

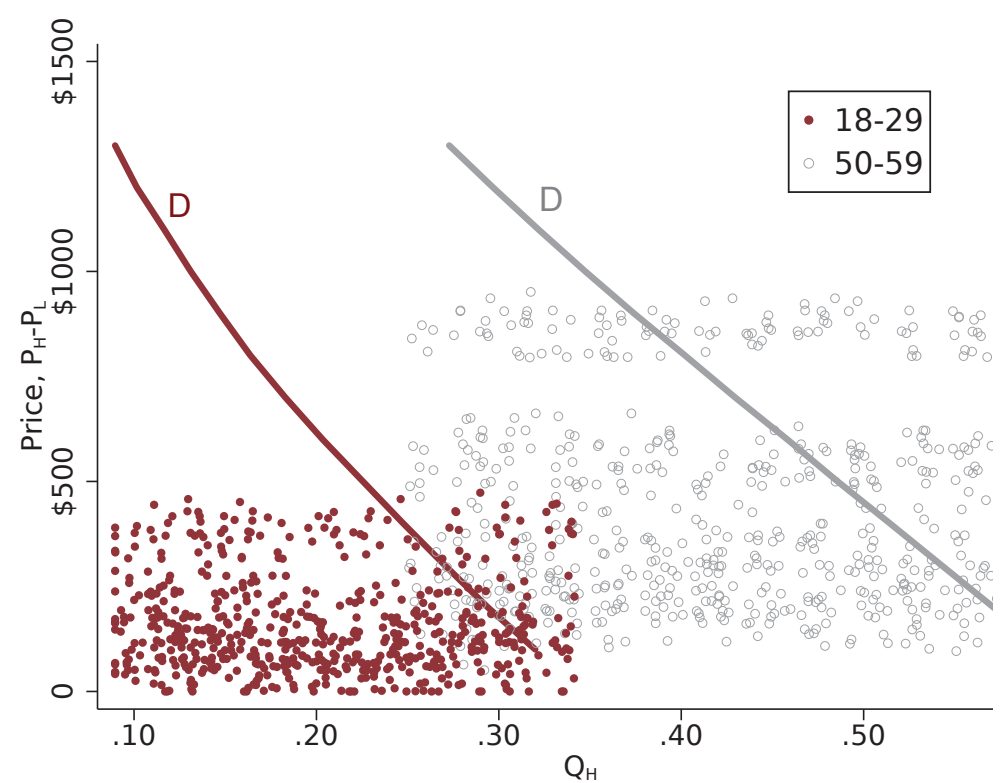

Note: Figure plots the empirical analogue of Figure 4. In Panel A, within- and across-type demand heterogeneity is represented in $(v, c)$ space. The dashed 45-degree line separates the cases in which enrolling in $H$ is socially efficient from those in which it is efficient to enroll in $L$. In Panel B, the corresponding demand and average cost clouds are plotted in $(\mathrm{P}, \mathrm{Q})$ space. In both panels, the insurer-borne healthcare costs, $c_{i j}$, are calculated directly from the medical risk distributions and cost-sharing rules, as described in Section 3.3. Consumer plan valuations are calculated using the utility function parameters from Table 6 to generate the certainty equivalent value of each plan for each consumer. Because the choice in this setting is between two plans (greater or lesser insurance), the relevant valuations and costs are the differences in valuations and costs between these plans: $v_{i \mathrm{H}}-v_{i \mathrm{~L}}$ and $c_{i \mathrm{H}}-c_{i \mathrm{~L}}$. See the text in Section 6.1 for additional details. 
Table 1: Cost-Sharing Rules for the Two Plans Available to Employees

\begin{tabular}{lcc}
\hline & $\begin{array}{c}\text { PPO Plan L } \\
(1)\end{array}$ & $\begin{array}{c}\text { PPO Plan H } \\
(2)\end{array}$ \\
\hline Deductible & $\$ 500$ & $\$ 300$ \\
Coinsurance & $20 \%$ & $10 \%$ \\
Out of Pocket Maximum & $\$ 4,250$ & $\$ 2,300$ \\
Copays & & \\
$\quad$ Emergency Dept & $\$ 0$ & $\$ 0$ \\
Well Visit & $\$ 0$ & $\$ 0$ \\
Drugs (brand/generic 30 day) & $\$ 10 / 5$ & $\$ 10 / 5$ \\
\hline
\end{tabular}

Note: Table lists cost-sharing rules for the two plans in employees' choice set. Both plans were PPOs with identical provider networks and differed only in cost-sharing rules. $H$ provides fuller financial insurance. The coinsurance rate is the marginal price faced by the consumer once the deductible has been met. Both the deductible and coinsurance are counted towards the out-of-pocket maximum. 
Table 2: Summary Statistics: Plan Choices and Average Claims Costs by Group

\begin{tabular}{|c|c|c|c|c|c|}
\hline & \multirow{2}{*}{$\begin{array}{c}\text { Fraction } \\
\text { Choosing } \\
\text { Plan H } \\
(1) \\
\end{array}$} & \multicolumn{3}{|c|}{ Average Healthcare Costs } & \multirow[b]{2}{*}{$\begin{array}{l}\text { Obs. } \\
(5)\end{array}$} \\
\hline & & $\begin{array}{c}\text { All Enrollees } \\
(2)\end{array}$ & $\begin{array}{c}\text { Conditional on } \\
\text { Plan L } \\
(3)\end{array}$ & $\begin{array}{c}\text { Conditional on } \\
\text { Plan H } \\
(4)\end{array}$ & \\
\hline Full Sample & 0.35 & $\$ 3,865$ & $\$ 2,672$ & $\$ 6,090$ & 22,299 \\
\hline \multicolumn{6}{|l|}{ By Age } \\
\hline $18-29$ & 0.22 & $\$ 1,820$ & $\$ 1,460$ & $\$ 3,094$ & 4,570 \\
\hline $30-39$ & 0.31 & $\$ 2,760$ & $\$ 2,103$ & $\$ 4,239$ & 5,787 \\
\hline $40-49$ & 0.37 & $\$ 4,355$ & $\$ 2,801$ & $\$ 6,957$ & 6,875 \\
\hline $50-59$ & 0.48 & $\$ 6,305$ & $\$ 4,958$ & $\$ 7,773$ & 5,067 \\
\hline \multicolumn{6}{|l|}{ By Sex } \\
\hline Men & 0.31 & $\$ 3,121$ & $\$ 2,193$ & $\$ 5,149$ & 13,550 \\
\hline Women & 0.40 & $\$ 5,017$ & $\$ 3,525$ & $\$ 7,223$ & 8,749 \\
\hline
\end{tabular}

Note: Table presents summary statistics on employees' plan choices and healthcare consumption for the main estimation sample. The sample consists of employees who enroll in either plan $L$ or $H$. Column (1) lists the fraction of enrollees who choose plan $H$. Average costs of healthcare consumed, measured as the total bills paid to service providers, are listed in columns (2) through (4). Column (2) lists the average expenditure overall. Columns (3) and (4) list expenditure conditional on the plan chosen. The first row lists these statistics for the entire estimation sample. The remaining rows repeat these statistics for different demographic sub-groups. 
Table 3: Main Result: Demand Heterogeneity Conditional on Expected Costs, $\widehat{C}_{i}$

\begin{tabular}{|c|c|c|c|c|c|}
\hline \multirow{2}{*}{$\begin{array}{l}\text { Dependent Variable: } \\
\text { Sample: }\end{array}$} & \multicolumn{5}{|c|}{ Chose Plan $\mathrm{H}$} \\
\hline & $\begin{array}{l}\text { Full } \\
\text { (1) }\end{array}$ & $\begin{array}{l}\text { Full } \\
\text { (2) }\end{array}$ & $\begin{array}{l}\text { Full } \\
\text { (3) }\end{array}$ & $\begin{array}{l}\text { Full } \\
(4)\end{array}$ & $\begin{array}{c}\text { Full } \\
\text { (5) }\end{array}$ \\
\hline \multirow[t]{2}{*}{ Expected cost, $\ln \left(\hat{C}_{t}\right)$} & 0.12 & 0.13 & 0.13 & 0.14 & 0.15 \\
\hline & $(0.01)$ & $(0.01)$ & $(0.01)$ & $(0.01)$ & $(0.01)$ \\
\hline \multirow[t]{2}{*}{ Age 30 to 39} & 0.05 & 0.05 & 0.05 & 0.05 & \\
\hline & $(0.01)$ & $(0.01)$ & $(0.01)$ & $(0.01)$ & \\
\hline \multirow[t]{2}{*}{ Age 40 to 49} & 0.06 & 0.05 & 0.05 & 0.05 & \\
\hline & $(0.01)$ & $(0.01)$ & $(0.01)$ & $(0.01)$ & \\
\hline \multirow[t]{2}{*}{ Age 50 to 59} & 0.10 & 0.10 & 0.09 & 0.09 & \\
\hline & $(0.01)$ & $(0.02)$ & $(0.02)$ & $(0.02)$ & \\
\hline \multirow[t]{2}{*}{ Female } & 0.02 & 0.02 & 0.03 & & 0.03 \\
\hline & $(0.01)$ & $(0.01)$ & $(0.01)$ & & (0.01) \\
\hline State FEs & & $x$ & $x$ & $x$ & $x$ \\
\hline Worker characteristics & & & $x$ & $x$ & $x$ \\
\hline Mean of dependent variable & 0.35 & 0.35 & 0.35 & 0.35 & 0.35 \\
\hline Observations & 22,299 & 22,299 & 22,299 & 22,299 & 22,299 \\
\hline
\end{tabular}

Note: Table reports results from a series of plan choice regressions that parallel the semi-parametric plots of Figure 6. These regressions correspond to the sufficient statistics test described in Equations (5) and (6). The dependent variable is an indicator for choosing the fuller insurance option, plan $H$. All regressions control for expected healthcare spending by including the natural log of the person-specific expected spending, which is predicted using the prior's years diagnoses and utilization. See Section 3 for full detail. Column (1) includes no additional controls. Columns (2) through (5) add state fixed effects and worker characteristics, which include indicators for hourly/salary, parttime/full-time, and union status. 18-29 is the excluded age category. Standard errors in parentheses are clustered at the person-level. 
Table 4: Robustness: No Correlation Between Age and Realized Costs, Conditional on $\widehat{C}_{i}$

\begin{tabular}{|c|c|c|c|c|c|c|}
\hline \multirow{2}{*}{$\begin{array}{l}\text { Dependent Variable: } \\
\text { Sample: }\end{array}$} & \multicolumn{6}{|c|}{ Realized Costs, $C_{t}$} \\
\hline & $\begin{array}{l}\text { Full } \\
(1)\end{array}$ & $\begin{array}{l}\text { Full } \\
(2)\end{array}$ & $\frac{\text { Age } 18-29}{(3)}$ & $\frac{\text { Age } 30 \text { to } 39}{(4)}$ & $\frac{\text { Age } 40 \text { to } 49}{(5)}$ & $\frac{\text { Age } 50 \text { to } 59}{(6)}$ \\
\hline Expected costs, $\hat{C}_{t}$ & $\begin{array}{c}1.13 \\
(0.07)\end{array}$ & $\begin{array}{c}1.12 \\
(0.08)\end{array}$ & $\begin{array}{c}1.29 \\
(0.23)\end{array}$ & $\begin{array}{c}0.98 \\
(0.11)\end{array}$ & $\begin{array}{c}1.14 \\
(0.11)\end{array}$ & $\begin{array}{c}1.13 \\
(0.14)\end{array}$ \\
\hline Intercept & $\begin{array}{l}\$ 8 \\
(201)\end{array}$ & $\begin{array}{l}-\$ 108 \\
(151)\end{array}$ & $\begin{array}{r}-\$ 394 \\
(318)\end{array}$ & $\begin{array}{l}\$ 290 \\
(234)\end{array}$ & $\begin{array}{l}\$ 114 \\
(311)\end{array}$ & $\begin{array}{c}-\$ 3 \\
(693)\end{array}$ \\
\hline Age 30 to 39 & & $\begin{array}{c}\$ 30 \\
(182)\end{array}$ & & & & \\
\hline Age 40 to 49 & & $\begin{array}{l}\$ 274 \\
(261)\end{array}$ & & & & \\
\hline Age 50 to 59 & & $\begin{array}{l}\$ 166 \\
(344)\end{array}$ & & & & \\
\hline F-stat on age variables & & $\begin{array}{c}0.45 \\
p=0.72\end{array}$ & & & & \\
\hline Observations & 22,299 & 22,299 & 4,570 & 5,787 & 6,875 & 5,067 \\
\hline
\end{tabular}

Note: Table reports results from a series of regressions of realized medical expenditure on predicted medical expenditure for the same plan year. These regressions test whether age has residual power to predict realized healthcare expenses after the expected cost measure for total spending is included as a control. Column (1) includes the single regressor indicated. Column (2) adds the same age and sex indicators as in Table 3. 18-29 is the excluded age category. Columns (3) through (6) run the regression in column (1) separately within subsamples defined by age group. Unbiased prediction would imply a slope not different from 1.0 and an intercept not different from 0 . Standard errors in parentheses are clustered at the person-level. 


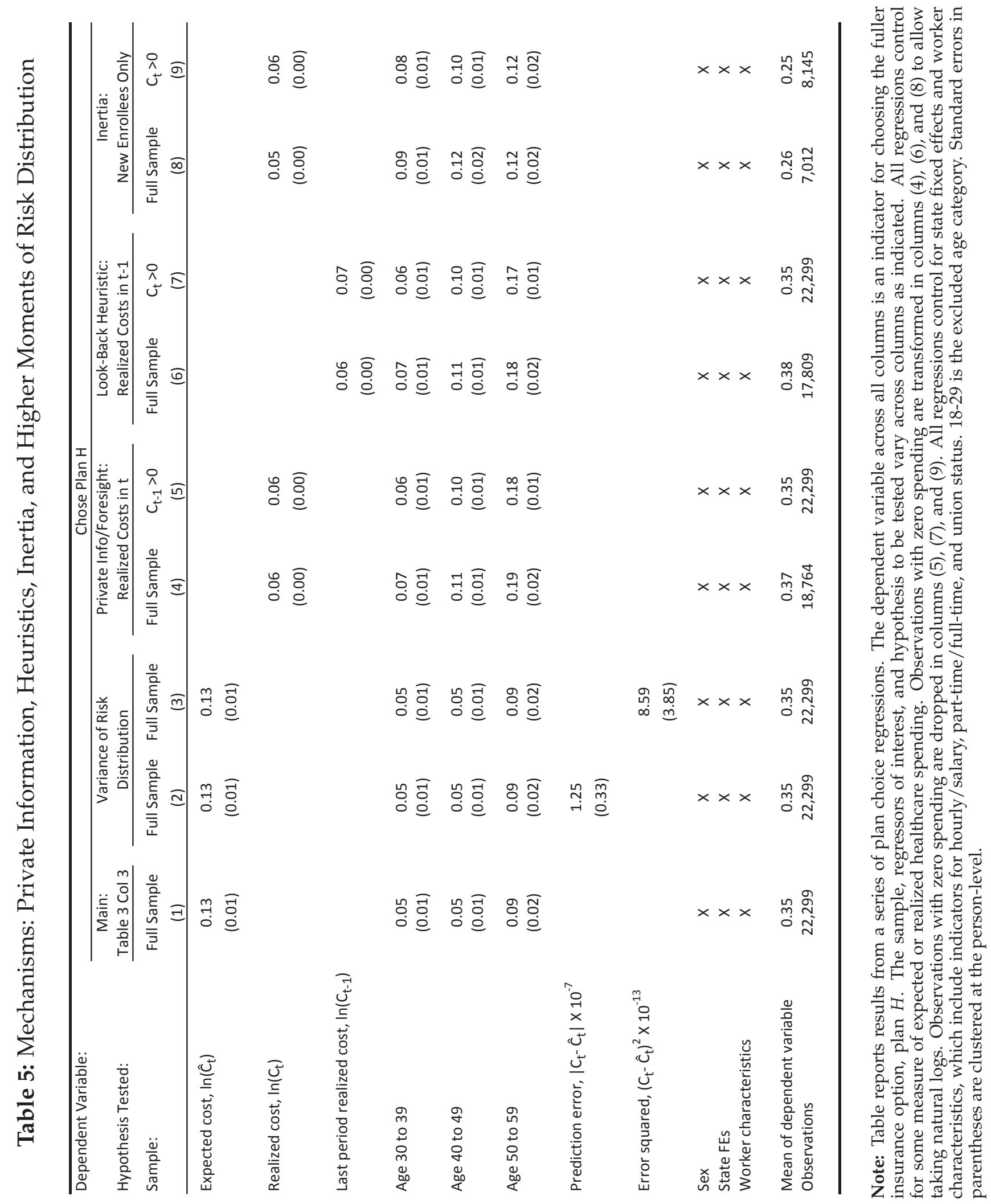


Table 6: SMLE Parameter Estimates from Expected Utility Model

\begin{tabular}{lcr}
\hline Parameter & Coefficient & SE \\
\hline Age 30 to 39 & $\$ 261$ & $(\$ 71)$ \\
Age 40 to 49 & $\$ 323$ & $(\$ 75)$ \\
Age 50 to 59 & $\$ 508$ & $(\$ 92)$ \\
Female & $\$ 178$ & $(\$ 50)$ \\
Plan H Intercept & $-\$ 832$ & $(\$ 153)$ \\
Coefficient of Absolute Risk Aversion & 0.000696 & $(0.000101)$ \\
Standard Deviation of $\varepsilon$ & $\$ 1,351$ & $(\$ 165)$ \\
\hline
\end{tabular}

Note: Results from the expected utility plan choice model. Standard errors are calculated from the inverse of the numerically-computed Hessian. See Section 5 for full detail.

Table 7: Risk Aversion Estimate in Context

\begin{tabular}{lcc}
\hline Reference & $\begin{array}{c}\text { Absolute Risk } \\
\text { Aversion }\end{array}$ & $\begin{array}{c}\text { Certainty } \\
\text { Equivalent }\end{array}$ \\
\hline Cohen-Einav (2007) median & $3.4 \times 10^{-5}$ & 99.7 \\
Metrick (1995) & $6.6 \times 10^{-5}$ & 99.3 \\
Gertner (1993) & $3.1 \times 10^{-4}$ & 97.0 \\
Handel (2010) median & $3.7 \times 10^{-4}$ & 95.2 \\
This Paper & $6.96 \times 10^{-4}$ & 93.5 \\
Handel (2010) mean & $7.9 \times 10^{-4}$ & 92.6 \\
Sydnor (2006) & $2.0 \times 10^{-3}$ & 83.3 \\
Cohen-Einav (2007) mean & $3.1 \times 10^{-3}$ & 76.5 \\
Holt and Laury (2002) & $3.2 \times 10^{-2}$ & 21.0 \\
\hline
\end{tabular}

Note: Table compares the estimated coefficient of absolute risk aversion from Table 6 to the literature. To aid interpretation, column (3) displays a certainty equivalent measure of the risk parameters. The certainty equivalent here is the amount $X$ that would make someone indifferent between accepting a gamble in which they win $\$ 100$ or lose $\$ X$ with equal probability versus a status quo where nothing happens. 
Table 8: Counterfactual Pricing and Welfare

\begin{tabular}{|c|c|c|c|c|c|}
\hline & \multirow{2}{*}{$\begin{array}{c}\text { Price } \\
\left(P_{H}-P_{L}\right) \\
(1)\end{array}$} & \multirow[b]{2}{*}{$\begin{array}{c}\text { Takeup } \\
\text { of } \mathrm{H} \\
(2)\end{array}$} & \multicolumn{3}{|c|}{ Relative to Baseline } \\
\hline & & & $\begin{array}{c}\text { Price } \\
\text { Change } \\
(3)\end{array}$ & $\begin{array}{c}\text { Takeup } \\
\text { Change } \\
(4)\end{array}$ & $\begin{array}{c}\text { Welfare } \\
\text { Change } \\
\text { (5) }\end{array}$ \\
\hline & \multicolumn{5}{|c|}{ Panel A: Baseline Case } \\
\hline Overall & $\$ 695$ & 0.311 & & & \\
\hline $18-29$ & $\$ 695$ & 0.184 & & & \\
\hline $30-39$ & $\$ 695$ & 0.279 & & & \\
\hline $40-49$ & $\$ 695$ & 0.338 & & & \\
\hline $50-59$ & $\$ 695$ & 0.430 & & & \\
\hline Women & $\$ 695$ & 0.274 & & & \\
\hline \multirow[t]{2}{*}{ Men } & $\$ 695$ & 0.376 & & & \\
\hline & \multicolumn{5}{|c|}{ Panel B: Optimal Uniform Pricing } \\
\hline Overall & $\$ 255$ & 0.427 & $-\$ 440$ & 0.116 & $\$ 24.57$ \\
\hline $18-29$ & $\$ 255$ & 0.282 & $-\$ 440$ & 0.098 & $\$ 31.66$ \\
\hline $30-39$ & $\$ 255$ & 0.394 & $-\$ 440$ & 0.114 & $\$ 29.02$ \\
\hline $40-49$ & $\$ 255$ & 0.459 & $-\$ 440$ & 0.121 & $\$ 22.90$ \\
\hline $50-59$ & $\$ 255$ & 0.556 & $-\$ 440$ & 0.126 & $\$ 14.98$ \\
\hline Women & $\$ 255$ & 0.386 & $-\$ 440$ & 0.112 & $\$ 27.57$ \\
\hline \multirow[t]{2}{*}{ Men } & $\$ 255$ & 0.497 & $-\$ 440$ & 0.121 & $\$ 19.34$ \\
\hline & \multicolumn{5}{|c|}{ Panel C: Optimal Age-Specific Pricing } \\
\hline Overall & & 0.428 & & 0.117 & $\$ 25.34$ \\
\hline $18-29$ & $\$ 145$ & 0.309 & $-\$ 550$ & 0.125 & $\$ 33.22$ \\
\hline $30-39$ & $\$ 220$ & 0.404 & $-\$ 475$ & 0.124 & $\$ 29.44$ \\
\hline $40-49$ & $\$ 270$ & 0.455 & $-\$ 425$ & 0.117 & $\$ 23.00$ \\
\hline \multirow[t]{2}{*}{$50-59$} & $\$ 350$ & 0.529 & $-\$ 345$ & 0.099 & $\$ 16.36$ \\
\hline & \multicolumn{5}{|c|}{ Panel D: Optimal Sex-Specific Pricing } \\
\hline Overall & & 0.429 & & 0.118 & $\$ 24.86$ \\
\hline Women & $\$ 210$ & 0.398 & $-\$ 485$ & 0.124 & $\$ 27.76$ \\
\hline Men & $\$ 310$ & 0.482 & $-\$ 385$ & 0.106 & $\$ 19.80$ \\
\hline
\end{tabular}

Note: Table reports plan enrollment and welfare under several counterfactual pricing scenarios. Panel A of Table 8 describes the baseline case of uniform premiums equal to $80 \%$ of the average insurer cost difference between plans. Panel B sets the single, non-discriminatory price for all enrollees that maximizes overall welfare subject to the constraint of a uniform price. Panel C sets the optimal age-specific prices. Panel D sets the optimal sex-specific prices. Columns (1) and (2) report the counterfactual price and quantity at that price. Columns (3) and (4) report the changes in price and quantity relative to baseline the in Panel A. Column (5) reports the corresponding welfare change. 


\section{Not For Publication}

\section{APPENDIX}

\section{A.1 Proof of Welfare-Improving Price Discrimination}

Suppose that there is both systematic preference heterogeneity over insurance (as discussed in Section 2 ) across groups defined by some characteristic $z$ as well as adverse selection within groups. Formally, $\operatorname{corr}(v, z \mid c) \neq 0$ and $\operatorname{corr}(v, c \mid z)>0$. If the group-specific demand functions are steeper than the marginal cost curves and exhibit single crossing, and if the efficient takeup rate of insurance is strictly between zero and one, then optimal prices differ across groups z-i.e., there is welfare improving price discrimination along the characteristic $z$, relative to the best uniform price.

Proof: Define $v_{a}(c)$ and $v_{b}(c)$ as willingness-to-pay for a fixed contract among consumers in groups $a$ and $b$ with costs $c$. Without loss of generality, define the group $z=a$ as the group for which willingness-to-pay conditional on costs is higher and define $z=b$ as the remainder of the market, so that $v_{a}(c)>v_{b}(c)$. The condition $\operatorname{corr}(v, c \mid z)>0$ implies that $v_{b}(c)$ and $v_{a}(c)$ are increasing in $c$.

Except for the degenerate cases where $v_{a}(c)>v_{b}(c)>c \forall c$ (all enrollees in the market efficiently insured) or where $c>v_{a}(c)>v_{b}(c) \forall c$ (all enrollees in the market efficiently uninsured), there exists some level of costs, $\underline{c}$, for which $v_{a}(\underline{c})>\underline{c}>v_{b}(\underline{c})$. That is, there exists some level of costs at which it is efficient to insure the $a$ types, but not the $b$ types. A simple version of this case that assumes linear $v(c)$ functions is displayed in Figure 2.

For group $a$ consumers with costs $\underline{c}$, only prices $p \leq \underline{c}$ sort efficiently. For group $b$ consumers with costs $\underline{c}$, only prices $p>\underline{c}$ sort efficiently. Therefore, no uniform price all consumers in both groups efficiently.

Now consider setting separate prices for groups $a$ and $b$. By the single-crossing assumption, within groups $a$ or $b$ all consumers can be sorted efficiently with the correct group-specific price. This within-group case is the standard case considered in the literature. (See, e.g., Einav and Finkelstein, 2011.) Therefore, optimal group-specific prices, which sort all consumers efficiently, are welfareimproving relative to any uniform price because no uniform price can sort all consumers with $\operatorname{costs} \underline{\underline{c}}$ efficiently.

\section{A.2 Worker and Firm Characteristics}

The firm is a manufacturer of consumer non-durables, with plant locations throughout the US. Files are purged of information that could be used to identify the employer or employees, though the anonymized employees are traceable across plan years. Workers are predominately male. Most are hourly. Workers who decline coverage are not in the data. Although no information is known regarding employees who waive coverage, data from the Kaiser Family Foundation (2007) Survey of Employer Health Benefits indicates that 15\% of workers in firms of similar size and in the same industry (consumer non-durables) around the study period waived coverage.

\section{A.3 SMLE Estimation Details for Section 6}

Simulated Maximum Likelihood Estimation (SMLE) is used to estimate Equation (8). Given the choice model outlined above, there is no closed-form expression for the choice probabilities. Therefore simulation is needed to calculate likelihood values. Here, I assume a normal error that enters the model non-linearly, but even if the error structure admitted a closed form solution to the choice 
probabilities (as would be the case, for example, with an additive extreme value error, which yields logit probabilities), simulation would still be necessary in order to numerically integrate over the risk distribution $F_{i j}(O O P)$.

Estimation begins by fixing the parameter vector $\theta$ and taking $Q$ draws from the distribution of out-of-pocket expenses, $F_{i j}(O O P)$, to calculate an expectation over health risk. Conditional on a set of values from the parameter vector (including a single draw of the error term $\epsilon_{i j}$ ), the expected utility for person $i$ in plan $j$ is:

$$
E\left[V_{i j}^{r}\right] \approx \frac{1}{Q} \sum_{q=1}^{Q}-e^{\gamma \cdot\left(P_{j}+O O P^{q}-\delta_{0} H-\sum_{a} \delta_{a} I_{a} \times H-\delta_{F} I_{F} \times H-\epsilon_{i j}^{r}\right)}
$$

The superscript $r$ denotes a draw from the normal error term.

A basic accept-reject (AR) simulator would assign a value of zero or one as the choice probability of plan $j$ depending on whether $j$ generated the highest utility given the simulated value of the error, $\epsilon_{i j}^{r}$. Here, I use smoothed AR simulator. For each draw $r$, a smoothing function is applied to ensure that the probability falls strictly between zero and one, avoiding problems otherwise arising from flat portions of the log likelihood function. I employ a logit smoothing kernel that follows Train (2009):

$$
S_{i j^{*}}^{r} \equiv \operatorname{Pr}\left(j=j^{*} \mid \theta^{r}, n\right)=\frac{\exp \left(E\left[V_{i j^{*}}^{r}\right] / \lambda\right)}{\sum_{J} \exp \left(E\left[V_{i j}^{r}\right] / \lambda\right)}
$$

This smoother approaches an unsmoothed AR simulator as $\lambda$ approaches zero. ${ }^{59}$ The output of the smoother is the probability of selecting a plan, conditional on values from the parameter vector, including a single draw from the normal error term. In order to calculate the simulated choice probability, many draws are taken from the error (still for a fixed parameter value of $\sigma_{\epsilon}$ ). Averaging the smoother values across these draws yields the simulated probability of person $i$ choosing plan $j$ :

$$
\breve{P}_{i j}(\theta) \equiv \frac{1}{R} \sum_{r=1}^{R} S_{i j}^{r}(\theta)
$$

Finally, the simulated log-likelihood function is defined by

$$
\operatorname{SLL}(\theta)=\frac{1}{N} \sum_{i=1}^{N} \sum_{j=1}^{J} d_{i j} \ln \breve{P}_{i j}(\theta)
$$

where $d_{i j}$ is an indicator function for the observed choice of each individual. Standard numerical maximization routines are used to search for the maximizing parameter vector. In practice, I set $Q=50, R=100$, and $\lambda>5$.

\footnotetext{
${ }^{59}$ The appearance of logit formula at this step has nothing to do with assumptions on the error term-it is purely a convenient way to ensure that choice probabilities fall strictly between zero and one, as in Train (2009).
} 
Figure A1: Model Implied by the Conventional Wisdom that Uniform Prices Can Sort Efficiently

(A) Homogeneity in $(v, c)$ Space

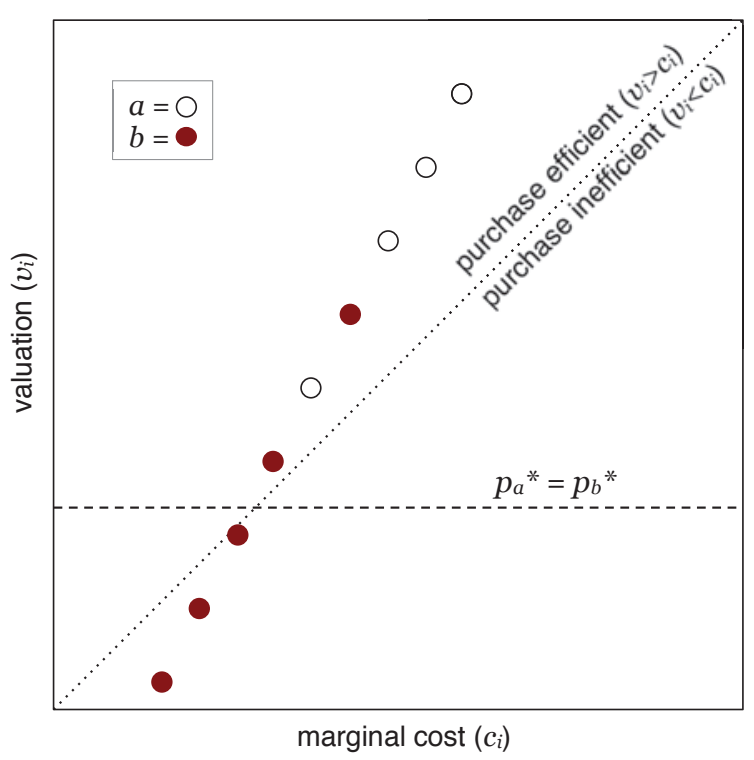

(B) Homogeneity in $(\mathrm{P}, \mathrm{Q})$ Space

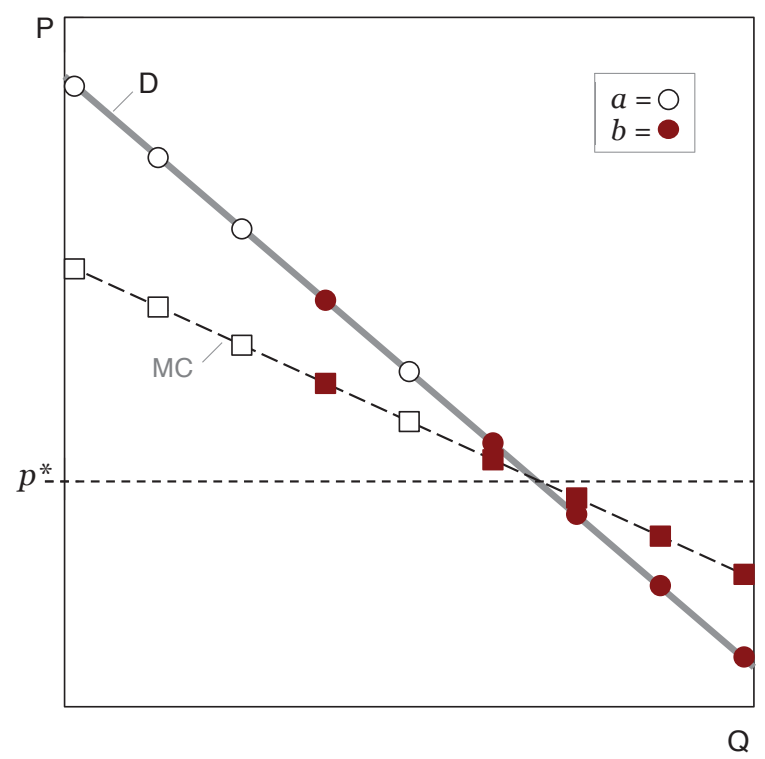

Note: Figure illustrates the model implicit in the conventional wisdom that uniform prices can achieve the feasible constrained optimal allocation. Here, a single price sorts efficiently, even though types differ in their costs on average. The view embodied in the figure implicitly relies on the assumption that consumer types differ only because they differ in the costs they generate, but not demand conditional on costs. See the notes to Figures 1 and 2 for additional documentation. 


\section{Figure A2: Expected Costs $\left(\widehat{C}_{i}\right)$ and Realized Costs $\left(C_{i}\right)$}

(A) Age 18-29

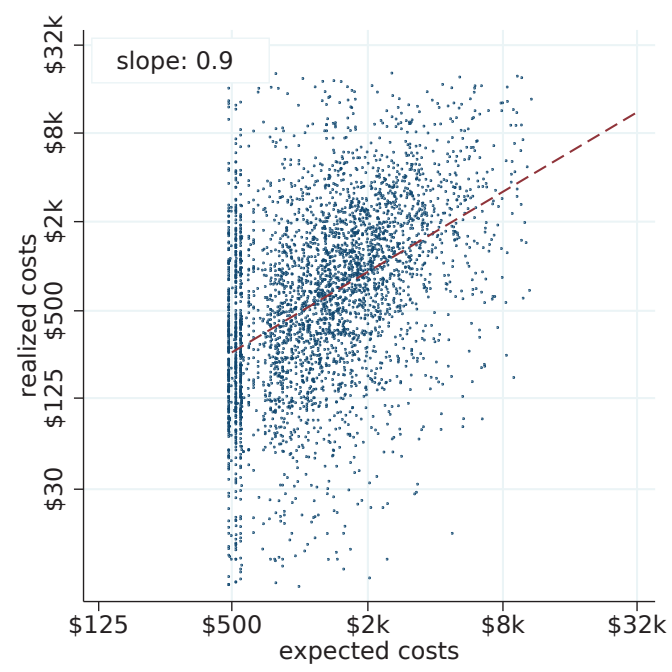

(C) Age 40-49

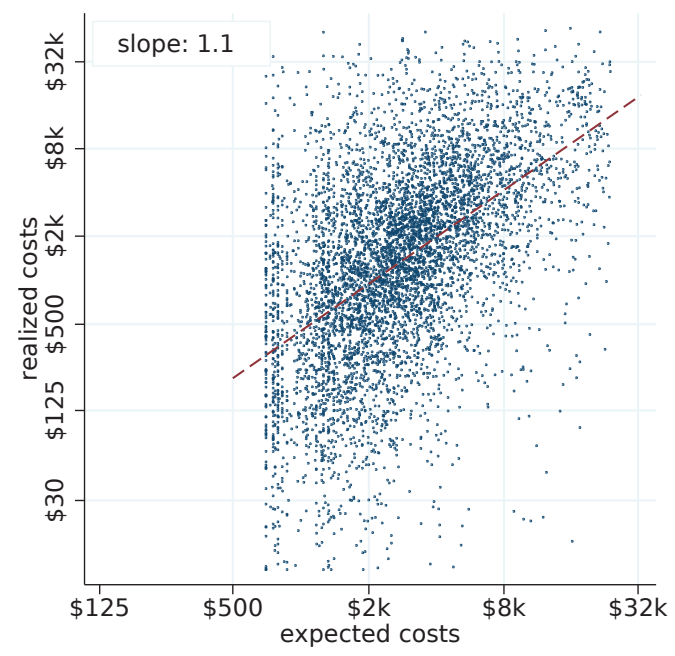

(B) Age 30-39

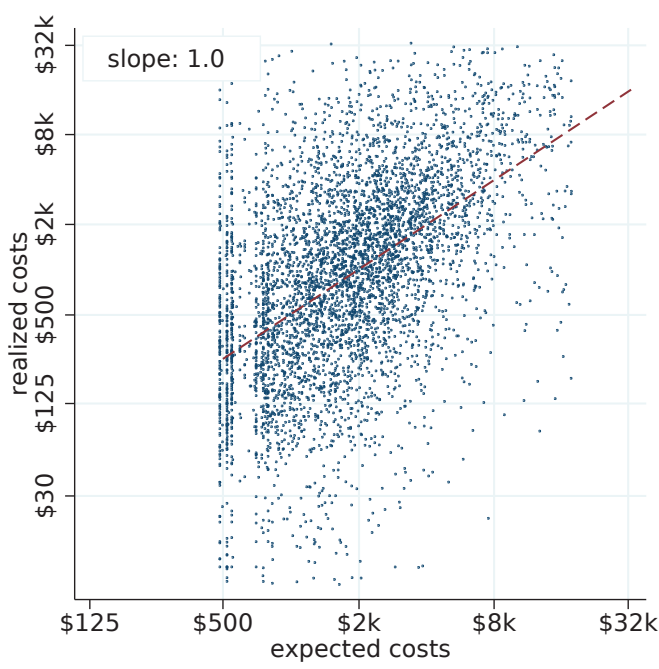

(D) Age 50-59

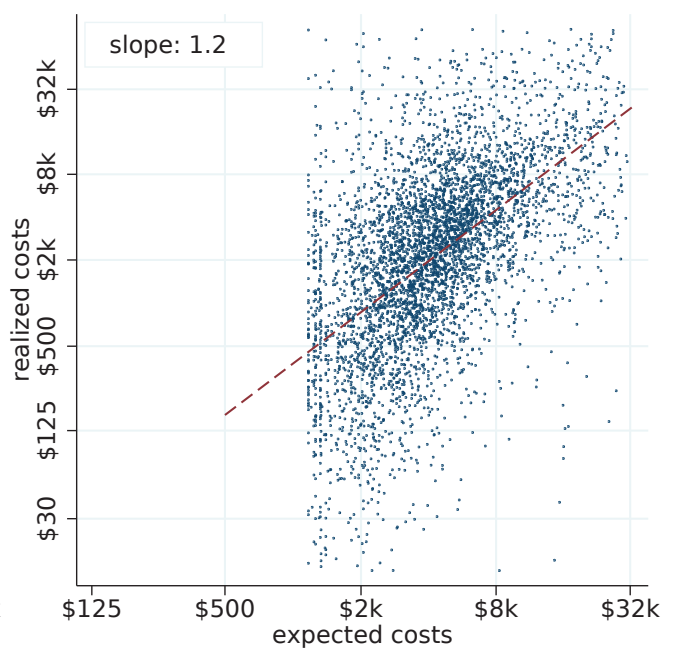

Note: Figure plots scatters of realized healthcare spending versus expected spending, including the insurer and consumer shares. Each panel contains a separate age group. Expected costs are predicted by the Johns Hopkins Adjusted Clinical Grouper (ACG) on the basis of prior year diagnoses and procedures. See text for full detail. The dashed line indicates the intercept and slope from a log-log regression of actual spending on expected spending. 
Figure A3: Robustness/Mechanisms: Demand Heterogeneity Using Alternative Cost Measures

(A) using ex-post realized costs in place of expected costs (perfect foresight approach)
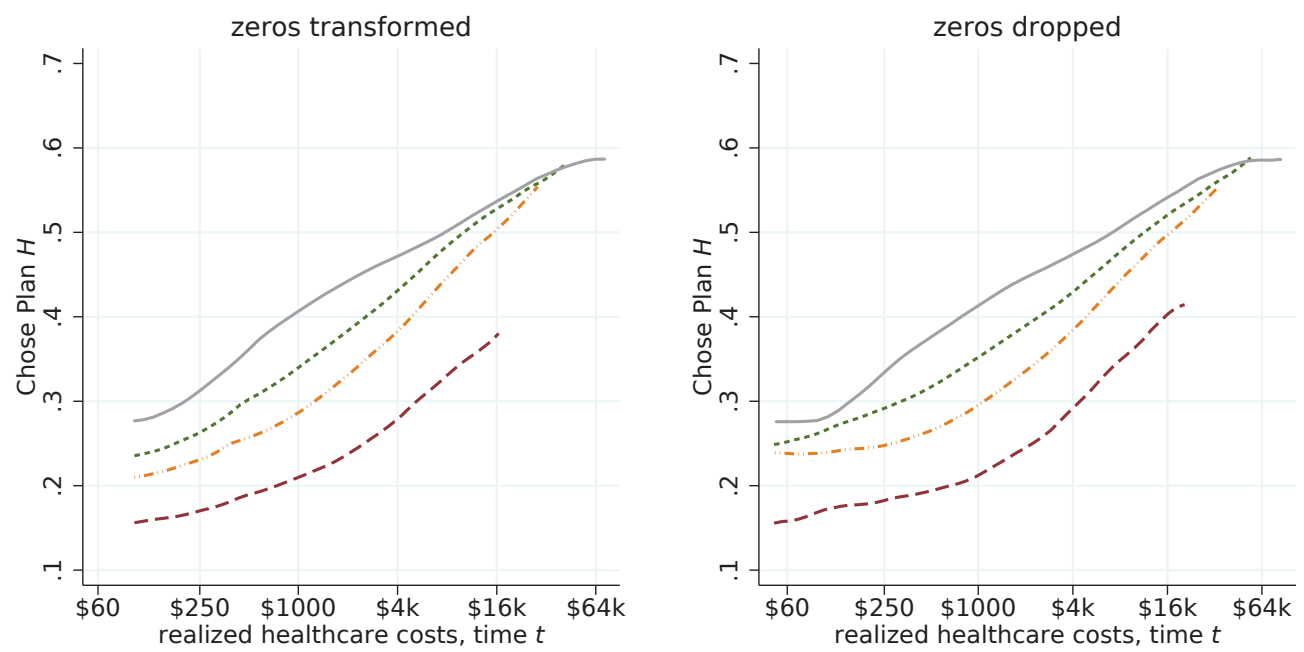

(B) using last year's costs in place of expected costs (heuristic approach)
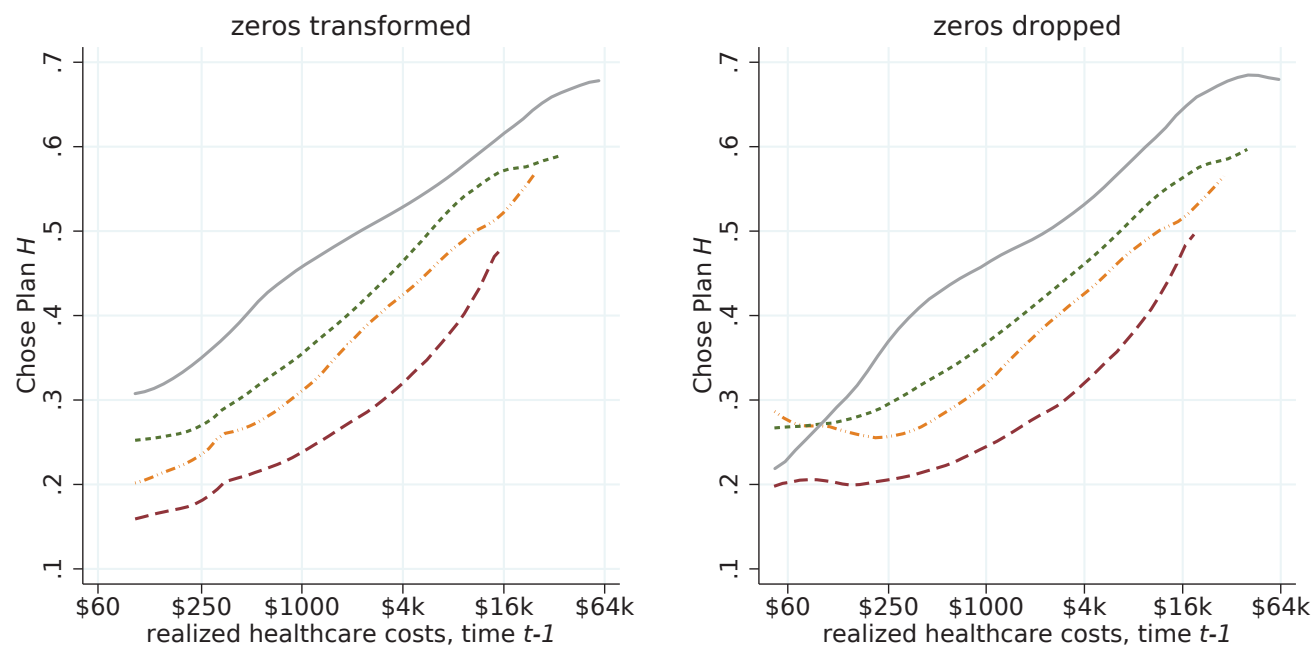

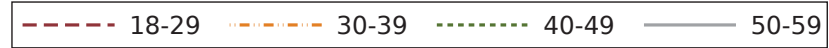

Note: Figure plots local polynomial regressions of plan choice against expected healthcare consumption. The dependent variable is an indicator for choosing the more generous plan, $H$. Each panel of the figure exactly replicates Panel A of Figure 6, except that along the horizontal axes, alternative cost measures are used. In Panel A, the current year's actual realized costs are the conditioning cost variable, rather than an unbiased measure of expected costs for the coming year. This would represent perfect foresight on the part of consumers, who choose plans prior to this realization. In Panel B, last year's actual costs are used as the conditioning variable. In the left panels, zeros are transformed to allow taking logs. In the right panels, zeroes are dropped from estimation. Consult the text and Figure 6 for additional detail. 
Figure A4: Model Fit: Simulated Plan Choices

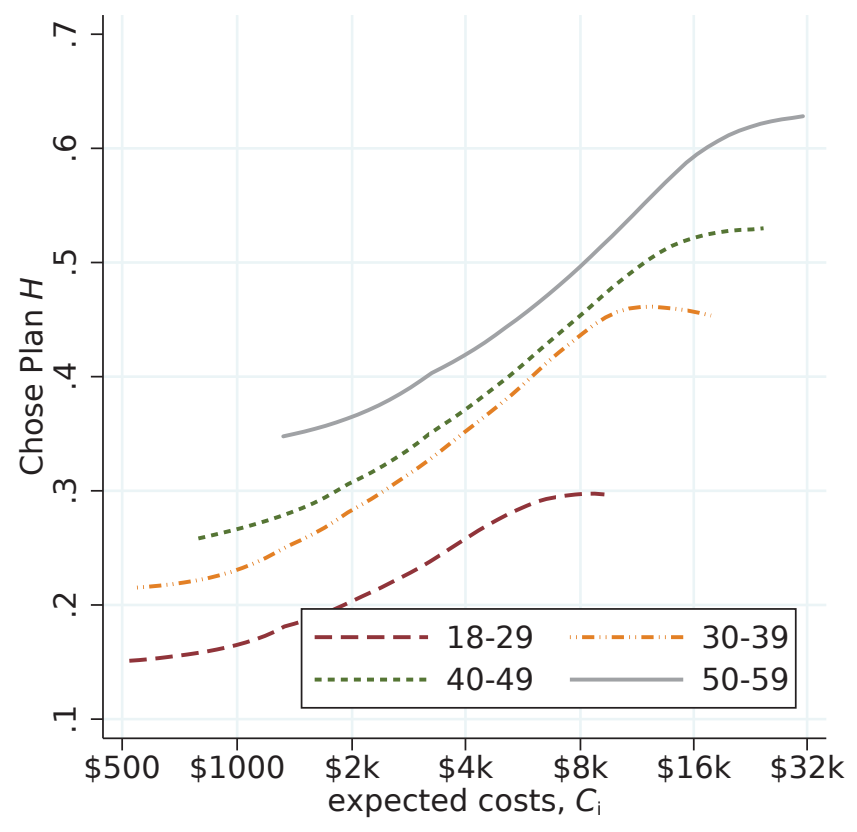

Note: Figure demonstrates the fit of the expected utility model. The plot replicates Panel of A of Figure 6 in plotting local polynomial regressions of plan choice on expected healthcare consumption. In this figure, rather than using the observed plan choice, the dependent variable is the simulated probability of choosing plan $H$. Simulated choices are predicted by the expected utility model parameters reported in Table 6. 